Australian Journal of

Crop Science

AJCS

AJCS 12(12):1962-1974 (2018)

ISSN:1835-2707

doi: 10.21475/ajcs.18.12.12.p1379

\title{
Nitric oxide enhances the adaptive responses of lupine plants against heavy-metal stress
}

\author{
Hanan A. Hashem* ${ }^{1}$, Amira A. Hassanein ${ }^{1}$, Nihal Y. Esmail ${ }^{2}$ \\ ${ }^{1}$ Department of Botany, Faculty of Science, Ain Shams University, Abbassia, Cairo, Egypt \\ ${ }^{2}$ National Institution of Occupational Safety and Health, Heliopolis, Cairo, Egypt
}

*Corresponding author: Hashem.hanan@gmail.com

\begin{abstract}
The present work investigates the possible protective role of nitric oxide (NO) against heavy metals stress in Lupinus termis L. plants. A greenhouse experiment was conducted as a $2 \times 2$ factorial design with five replications. Lupine seedlings were treated with two concentrations ( $0.4 \mathrm{mM}$ and $0.6 \mathrm{mM}$ ) of sodium nitroprusside (SNP as an NO donor) and subjected to high levels of nickel (Ni) sulfate (100 and $150 \mathrm{mM}$ ). The toxic effects of $\mathrm{Ni}$ on $\mathrm{L}$. termis plants were evaluated by measuring the oxidative stress markers such as lipid peroxidation, hydrogen peroxide and electrolyte leakage. In addition, growth parameters, endogenous phytohormones, photosynthetic pigment, calcium, magnesium and $\mathrm{Ni}$ accumulation in lupin plants grown under $\mathrm{Ni}$ toxicity in the presence or absence of NO were also determined. Carbohydrate, organic acid and proline contents were also measured to determine the possible NO-mediated defense strategies in lupine plants to resist $\mathrm{Ni}$ stress. The obtained results proved that, $\mathrm{Ni}$ toxicity resulted in significantly elevated levels of oxidative stress markers, as well as abscisic acid, jasmonic acid and proline contents, which are associated with significant reduction in growth parameters, photosynthetic pigments, carbohydrate, indole acetic acid and gibberellic acid contents compared with control plants. SNP treatments significantly alleviated the toxic effect of $\mathrm{Ni}$ on Lupinus termis $\mathrm{L}$. and increased the amounts of proline, soluble sugars and polysaccharides in shoots and roots, which could be an induced defensive mechanism against heavy-metal stress. Treatment with $0.4 \mathrm{mM}$ SNP was more effective in increasing lupine plants tolerance to Ni toxicity than the $0.6 \mathrm{mM} \mathrm{SNP}$.
\end{abstract}

Keywords: Lupinus termis; nickel, oxidative markers; phytohormones; sodium nitroprusside.

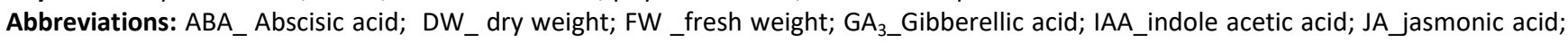
MDA_malondialdehyde; NED_N-naphthyl-ethylenediamine; NO_nitric oxide; NOS_nitric oxide synthase; ROS_reactive oxygen species; SNP_sodium nitroprusside; TBA_thiobarbituric acid ; TCA_trichloroacetic.

Introduction

Nitrogen monoxide or nitric oxide (NO) is a unique ubiquitous signaling molecule in animals and plants (Wendehenne et al., 2001). NO is produced in plants through different pathways: enzymatically by nitrate reductase, peroxidases and/or xanthine oxidoreductases and putative nitric oxide synthases; and non-enzymatically through, for example, nitrite reduction by ascorbic acid at a low $\mathrm{pH}$ or by carotenoids in light (Wendehenne et al., 2001; Belligni et al., 2001; Wendehenne et al., 2004; Desikan et al., 2004; Zemojtel et al., 2006). NO also plays vital roles in diverse physiological functions in plants, such as the induction of seed germination and reduction of seed dormancy (Beligni and Lamattina 2000; Bethke et al., 2006, 2007; Libourel et al., 2006; Zheng et al., 2009), regulation of plant metabolism and senescence (Guo and Crawford, 2005), induction of cell death (Pedroso and Durzan, 2000), regulation of stomatal movement (García-Mata and Lamattina, 2001; Guo et al., 2003; Neill et al., 2003; Sakihama et al., 2003; Bright et al., 2006; García-Mata and Lamattina, 2007), regulation of photosynthesis (Takahashi and Yamasaki
2002) and the regulation of flowering (He et al., 2004), as well as in mitochondrial functionality (Zottini et al. 2002) and gravitropism (Hu et al., 2005). It is believed that NO can tightly interact with the plant hormonal system and may serve as a secondary messenger (Lamattina et al., 2003).

In addition to its regulatory roles in plant development, NO has been implicated in plant responses to a variety of abiotic and biotic stresses, such as drought (García-Mata and Lamattina, 2002), salt (Zhang et al., 2006a, b; 2007), heat (Uchida et al. 2002) and infection (Modolo et al. 2005; Floryszak-Wieczorek et al., 2007). Both biotic and abiotic stresses alter (promote or suppress) NO production, while externally applied NO donors enhance plant tolerance to specific stresses (García-Mata and Lamattina, 2002; Uchida et al., 2002; Zhao et al., 2007).

Although heavy metals are normal constituents of soils and occur naturally in the environment, the contamination of soils by toxic metals and metalloids is of major concern worldwide (Rascio and Navari-Izzo, 2011; Villiers et al., 2011). Their toxic influence on plants is largely a strong and fast inhibition of the 
growth processes of the above- and underground parts, together with a decreased activity level of the photosynthetic apparatus, which is often correlated with progressing senescence-related processes (Molas, 2002; Sobkowiak and Dekert, 2003; Alaoui-Sossé et al., 2004; Lin et al., 2005). Growth inhibition and senescence stimulation, resulting from an excess of heavy metals are important effects (Maksymiec, 2007). In higher plants, heavy metals induce oxidative stress by the generation of superoxide radicals, hydrogen peroxide $\left(\mathrm{H}_{2} \mathrm{O}_{2}\right)$, hydroxyl radicals and singlet oxygen, collectively termed as reactive oxygen species (ROS). ROS can rapidly attack all types of bio-molecules, such as nucleic acids, proteins and amino acids, which lead to irreparable metabolic dysfunction and cell death. ROS produced under stress are detrimental to growth because these molecules cause gradual lipid peroxidation and the inactivation of antioxidant enzymes (Teisseire and Guy, 2000). NO is a highly reactive molecule and, as a free radical, it scavenges other reactive intermediates. It can alleviate the oxidative stress in plants generated by abiotic stresses (Kopyra and Gwozdz, 2003; Neill et al. 2003; Guo and Crawford, 2005).

The present work investigated the capability of NO to protect Lupinus termis plants against nickel (Ni) stress and highlighted the defense mechanism(s) underlying the NO-induced tolerance.

\section{Results}

\section{Endogenous NO content}

In general, lupine plants accumulate higher amounts of NO in their roots than shoots. An increase in endogenous NO content was observed in both shoots and roots of L. termis when treated with $0.4 \mathrm{mM}$ and $0.6 \mathrm{mM} \mathrm{SNP}$, compared with water as the control. The amount of NO was directly proportional to the applied concentration of SNP.

Treatment with Ni sulfate (100 and $150 \mathrm{mM}$ ) caused significant increases in the NO content. The maximum increase was detected in response to $150 \mathrm{mM}$ Ni sulfate and was calculated at $142.7 \%$ and $106.67 \%$ above the control value in shoots and roots, respectively (Table 1). Plants treated with both $150 \mathrm{mM}$ Ni sulfate + 0.6 mM SNP produced the highest amounts of NO in both shoots and roots, compared with other treated plants. The increase was evaluated as $24.78 \%$ and $4.03 \%$ in shoot and $10.51 \%$ and $40.31 \%$ in root over those treated with $150 \mathrm{mM} \mathrm{Ni}$ sulfate or $0.6 \mathrm{mM}$ SNP alone, respectively.

\section{Growth parameters}

Data illustrated in Table 2 indicated that the effect of exogenous NO applications on plant growth depended on the SNP concentration applied. The 0.4-mM SNP treatment caused a significant increase in all of the measured growth parameters, while the $0.6-\mathrm{mM}$ SNP treatment decreased almost all of the measured growth parameters, including a significant reduction $(P<0.05)$ in shoot and root lengths calculated at $10.31 \%$ and $18.08 \%$, respectively, less than control plants.
Ni toxicity (100 and $150 \mathrm{mM}$ ) dramatically reduced lupine growth. The detected toxic effect on growth was concentration dependent. The reduction in the measured growth parameters ranged from significant $(P<0.05)$ to extremely significant $(P<0.001)$ compared with the control values. Root growth in lupine was more affected than shoot growth by $\mathrm{Ni}$ stress, as shown by the $37.5 \%$ and $46.9 \%$ reductions in root length and root $\mathrm{FW}$, respectively, in plants that received $150 \mathrm{mM}$ Ni sulfate compared with control values. The growth of lupine plants treated with both SNP and $\mathrm{Ni}$ sulfate were less than those treated with the same concentration of SNP alone and greater than those treated with the corresponding concentration of $\mathrm{Ni}$ sulfate (Table 2).

An exogenous application of $0.4 \mathrm{mM}$ SNP completely alleviated the inhibitory effect of $100 \mathrm{mM}$ Ni sulfate on lupine growth.

\section{Phytohormonal contents}

The effects of SNP and $\mathrm{Ni}$ sulfate on the endogenous phytohormonal contents of lupine shoots and roots are illustrated in Table 3. Treatments with $0.4 \mathrm{mM}$ SNP caused extremely significant increases in the endogenous pool of phytohormones (including IAA, $G_{3}, A B A$ and $J A$ ) in lupine plants. Such increases ranged from $11.76 \%$ for IAA to $18.62 \%$ for JA contents of shoots treated with $0.4 \mathrm{mM} \mathrm{SNP}$, compared with control untreated plants. This stimulatory effect was pronounced in both shoots and roots. In contrast, the higher SNP concentration $(0.6 \mathrm{mM})$ and both applied $\mathrm{Ni}$ sulfate concentrations (100 and $150 \mathrm{mM}$ ) had inhibitory effects on plant hormone levels. The maximum inhibition of endogenous phytohormones was detected in plants receiving $150 \mathrm{mM} \mathrm{Ni}$ sulfate and was evaluated in lupine shoots as $32.35 \%, 62.88 \%$, $69.23 \%$ and $58.62 \%$ and in roots as $62.96 \%, 57.14 \%, 54.54 \%$ and $77.78 \%$ of $\mathrm{IAA}, \mathrm{GA}_{3}, \mathrm{ABA}$ and $\mathrm{JA}$, respectively, compared with control values.

\section{Photosynthetic pigment contents}

Data presented in Table 4 show that SNP treatments $(0.4$ and $0.6 \mathrm{mM}$ ) have positive impacts on photosynthetic pigment accumulation in lupine plants. Chlorophyll a underwent a highly significant increase compared with in untreated control plants. In contrast, Ni stress (100 mM and $150 \mathrm{mM}$ ) caused the significant to extremely significant inhibitions of chlorophyll a and $b$, carotenoids and total pigment content when compared with those of the control. These inhibition levels were directly proportional to the applied concentration of $\mathrm{Ni}$ sulfate and were calculated as $79.47 \%, 62.04 \%, 79.27 \%$ and $75.82 \%$ lower in plants receiving $150 \mathrm{mM} \mathrm{Ni}$ sulfate for chlorophyll a, chlorophyll b, carotenoids and total pigment content, respectively, than in the untreated control plants. Treatment with $0.4 \mathrm{mM}$ SNP partially alleviated the inhibitory effect of $\mathrm{Ni}$ toxicity on the lupine pigment content. 
Table 1. Effect of exogenous application of SNP (as NO donor) on nitric oxide content of Lupinus termis L. under Ni stress (0, 100 and $150 \mathrm{mM})$.Results are expressed in $\mu \mathrm{g} \mathrm{g}^{-1}$ fresh weight. Data are mean \pm SE (Standard Error) of five replicates.

\begin{tabular}{|c|c|c|c|c|c|c|c|}
\hline \multicolumn{2}{|l|}{ Treatment } & \multicolumn{3}{|c|}{ Shoot } & \multicolumn{3}{|c|}{ Root } \\
\hline \multirow{3}{*}{ Control } & $\mathrm{H}_{2} \mathrm{O}$ & 155.22 & \pm & 0.87 & 242.55 & \pm & 0.83 \\
\hline & SNP $0.4 \mathrm{mM}$ & 302.15 & \pm & $2.19^{c}$ & 380.79 & \pm & $0.82^{\mathrm{c}}$ \\
\hline & SNP $0.6 \mathrm{mM}$ & 451.91 & \pm & $0.08^{\mathrm{c}}$ & 394.79 & \pm & $0.08^{\mathrm{C}}$ \\
\hline Nickel sulphate & $100 \mathrm{mM}$ & 330.16 & \pm & $11.57^{b}$ & 372.35 & \pm & $12.99^{\mathrm{a}}$ \\
\hline Nickel sulphate $100 \mathrm{mM}+\mathrm{SNP} 0.4 \mathrm{mM}$ & & 359.53 & \pm & $1.70^{* * *}$ & 399.96 & \pm & 5.48 \\
\hline Nickel sulphate $100 \mathrm{mM}+\mathrm{SNP} 0.6 \mathrm{mM}$ & & 379.77 & \pm & $10.22^{*}$ & 418.29 & \pm & 6.29 \\
\hline Nickel sulphate & $150 \mathrm{mM}$ & 376.75 & \pm & $12.67^{b}$ & 501.28 & \pm & $1.15^{\mathrm{c}}$ \\
\hline Nickel sulphate $150 \mathrm{mM}+\mathrm{SNP} 0.4 \mathrm{mM}$ & & 427.07 & \pm & $13.77^{*}$ & 510.70 & \pm & $2.08^{* * *}$ \\
\hline Nickel sulphate $150 \mathrm{mM}+\mathrm{SNP} 0.6 \mathrm{mM}$ & & 470.11 & \pm & 64.60 & 553.94 & \pm & $4.40^{* * 4}$ \\
\hline
\end{tabular}

Values with a superscript are significantly different from the control (tap water). Letter "a" at P<0.05 (significant), "b" at P<0.01 (highly significant), "c" at P<0.001 (extremely significant), and the absence of letter = non-significant. Values with a superscript are significantly different from the control (SNP $0.4 \mathrm{mM}$, SNP $0.6 \mathrm{mM}$ ). Asterisk * at $\mathrm{P}<0.05$ (significant), ** at $\mathrm{P}<0.01$ (highly significant), ****at $\mathrm{P}<0.001$ (extremely significant), and the absence of letter $=$ non-significant.

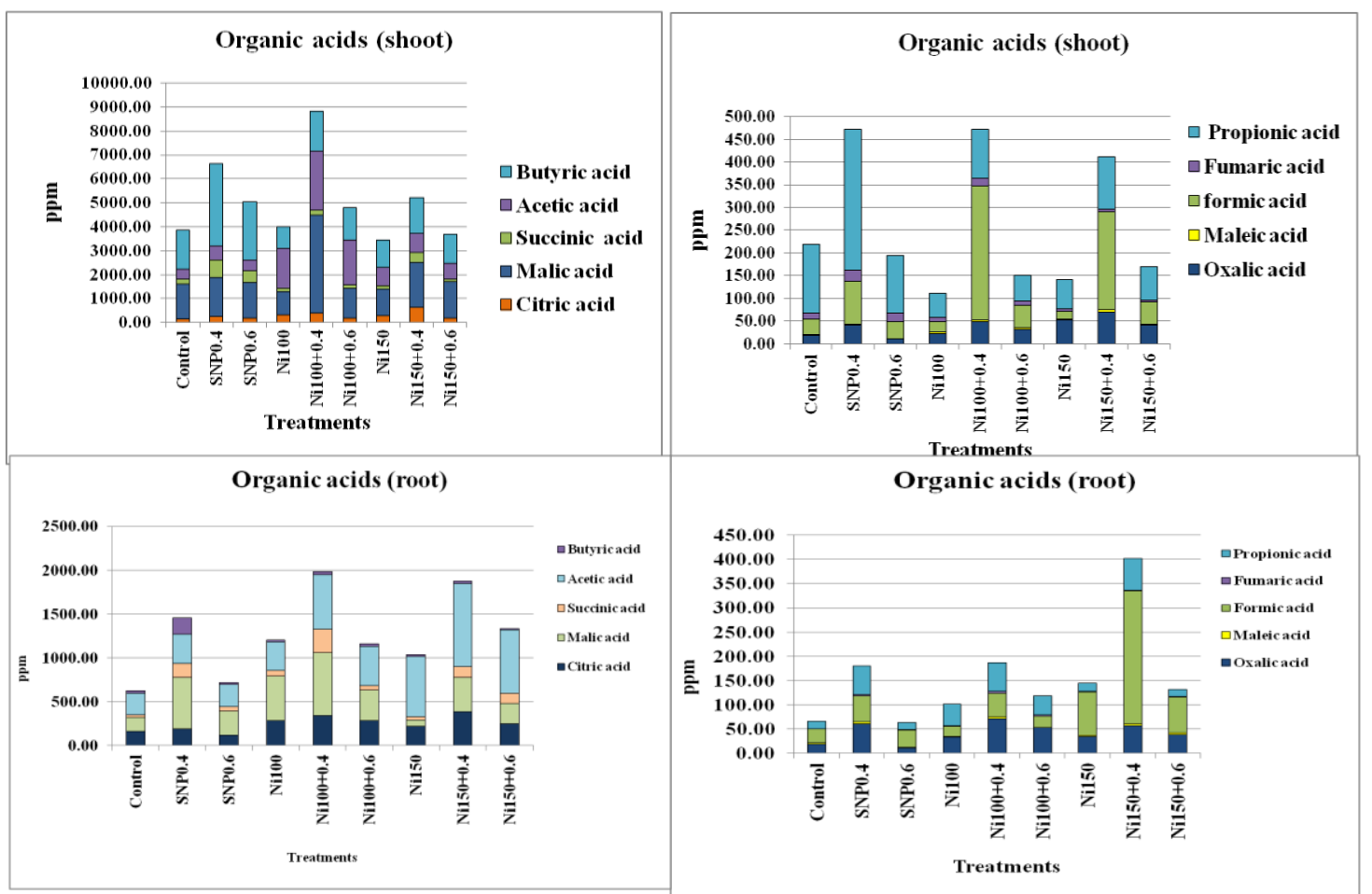

Fig 1. Effect of exogenous application of SNP (as NO donor), on organic acids of shoots and roots of Lupinus termis L. under Ni stress (0, 100 and 150 mM). 
Table 2. Effect of exogenous application of SNP (as NO donor) on growth characteristics of Lupinus termis L. under Ni stress (0, 100 and 150 mM). Data are mean \pm SE (Standard Error) of five replicates.

\begin{tabular}{|c|c|c|c|c|c|c|c|c|c|c|c|c|c|c|c|c|c|c|c|c|c|c|c|c|c|}
\hline \multirow{2}{*}{$\begin{array}{l}\text { Treatment } \\
\text { Control }\end{array}$} & \multirow{2}{*}{$\begin{array}{l}\begin{array}{l}\text { Growth } \\
\text { parameters }\end{array} \\
\mathrm{H}_{2} \mathrm{O}\end{array}$} & \multicolumn{3}{|c|}{$\begin{array}{l}\text { Shoot Length } \\
(\mathrm{cm})\end{array}$} & \multicolumn{3}{|c|}{$\begin{array}{l}\text { Root Length } \\
(\mathrm{cm})\end{array}$} & \multicolumn{3}{|c|}{$\begin{array}{c}\text { no. of } \\
\text { leaves/plant }\end{array}$} & \multicolumn{3}{|c|}{$\begin{array}{l}\text { area of leaves } \\
\left(\mathrm{cm}^{2}\right)\end{array}$} & \multicolumn{3}{|c|}{$\begin{array}{l}\text { fresh wt. of } \\
\text { shoot } \\
\text { (g) }\end{array}$} & \multicolumn{3}{|c|}{$\begin{array}{l}\text { dry wt. of shoot } \\
\text { (g) }\end{array}$} & \multicolumn{3}{|c|}{$\begin{array}{l}\text { fresh wt. of root } \\
\text { (g) }\end{array}$} & \multicolumn{3}{|c|}{$\begin{array}{l}\text { dry wt. of root } \\
\text { (g) }\end{array}$} \\
\hline & & 13.97 & \pm & 0.03 & 12.00 & \pm & 0.50 & 13.33 & \pm & 0.33 & 66.99 & \pm & 6.41 & 4.36 & \pm & 0.00 & 0.49 & \pm & 0.00 & 1.94 & \pm & 0.06 & 0.33 & \pm & 0.02 \\
\hline & SNP $0.4 \mathrm{mM}$ & 14.53 & \pm & $0.03^{c}$ & 13.67 & \pm & 0.44 & 16.00 & \pm & $0.00^{\mathrm{a}}$ & 73.42 & \pm & 2.40 & 4.65 & \pm & $0.07^{\mathrm{a}}$ & 0.61 & \pm & $0.01^{\mathrm{a}}$ & 2.23 & \pm & 0.11 & 0.42 & \pm & $0.01^{\mathrm{a}}$ \\
\hline & SNP $0.6 \mathrm{mM}$ & 12.53 & \pm & $0.03^{\mathrm{c}}$ & 9.83 & \pm & $0.17^{\mathrm{a}}$ & 13.00 & \pm & 0.00 & 67.06 & \pm & 3.22 & 4.24 & \pm & 0.15 & 0.54 & \pm & 0.01 & 1.88 & \pm & 0.02 & 0.31 & \pm & 0.03 \\
\hline Nickel sulphate & $100 \mathrm{mM}$ & 12.53 & \pm & $0.03^{\mathrm{c}}$ & 9.17 & \pm & $0.33^{\mathrm{a}}$ & 12.00 & \pm & 0.00 & 56.10 & \pm & 3.08 & 3.91 & \pm & $0.06^{\mathrm{a}}$ & 0.42 & \pm & $0.00^{c}$ & 1.34 & \pm & $0.02^{b}$ & 0.29 & \pm & 0.00 \\
\hline \multicolumn{2}{|c|}{ Nickel sulphate $100 \mathrm{mM}$} & 14.17 & \pm & 0.60 & 11.17 & \pm & $0.33^{*}$ & 13.33 & \pm & $0.33^{*}$ & 68.61 & \pm & 3.68 & 4.33 & \pm & 0.13 & 0.50 & \pm & 0.04 & 1.87 & \pm & 0.40 & 0.41 & \pm & 0.01 \\
\hline \multirow{2}{*}{\multicolumn{2}{|c|}{$\begin{array}{l}\text { +SNP0.4mM } \\
\text { Nickel sulphate } 100 \mathrm{mM}\end{array}$}} & & & & & & & & & & & & & & & & & & & & & & & & \\
\hline & & 11.50 & \pm & $0.00^{* * *}$ & 8.83 & \pm & $0.17^{*}$ & 11.67 & \pm & 0.33 & 65.05 & \pm & 4.29 & 3.86 & \pm & 0.19 & 0.49 & \pm & 0.03 & 1.44 & \pm & $0.02^{* * *}$ & 0.35 & \pm & 0.01 \\
\hline \multicolumn{26}{|c|}{ +SNP0.6mM } \\
\hline \multicolumn{2}{|c|}{ Nickel sulphate $150 \mathrm{mM}$} & 11.33 & \pm & $0.16^{* *}$ & 10.33 & \pm & $0.16^{*}$ & 12.67 & \pm & $0.33^{*}$ & 51.86 & \pm & $7.19^{*}$ & 3.82 & \pm & 0.28 & 0.50 & \pm & 0.03 & 1.67 & \pm & $0.04^{*}$ & 0.39 & \pm & 0.01 \\
\hline \multicolumn{2}{|c|}{$\begin{array}{l}\text { +SNP0.4mM } \\
\text { Nickel sulphate } 150 \mathrm{mM} \\
+ \text { +SNP0.6mM }\end{array}$} & 10.00 & \pm & $0.50^{*}$ & 8.17 & \pm & $0.16^{* *}$ & 11.67 & \pm & 0.67 & 49.12 & \pm & $0.88^{*}$ & 3.59 & \pm & 0.21 & 0.46 & \pm & $0.01^{*}$ & 1.27 & \pm & $0.01^{* * *}$ & 0.33 & \pm & 0.02 \\
\hline
\end{tabular}

Table 3. Effect of exogenous application of SNP (as NO donor) on acidic hormones (IAA, GA $\mathrm{A}_{3}$ and ABA) and JA content of Lupinus termis $\mathrm{L}$. under Ni stress ( 0 , 100 and 150 mM). Results are expressed in ppm fresh weight. Data are mean \pm SE (Standard Error) of five replicates.

\begin{tabular}{|c|c|c|c|c|c|c|c|c|c|c|c|c|c|c|c|c|c|c|c|c|c|c|c|c|c|}
\hline \multirow{3}{*}{ Treatment } & \multirow{3}{*}{$\frac{\text { Conc. }}{\left(\mathrm{H}_{2} \mathrm{O}\right)}$} & \multicolumn{6}{|l|}{ IAA } & \multicolumn{6}{|l|}{$\mathrm{GA}_{3}$} & \multicolumn{6}{|l|}{ ABA } & \multicolumn{6}{|l|}{ JA } \\
\hline & & \multicolumn{3}{|c|}{ Shoot } & \multicolumn{3}{|c|}{ Root } & \multicolumn{3}{|c|}{ Shoot } & \multicolumn{3}{|c|}{ Root } & \multicolumn{3}{|c|}{ Shoot } & \multicolumn{3}{|c|}{ Root } & \multicolumn{3}{|c|}{ Shoot } & \multicolumn{3}{|c|}{ Root } \\
\hline & & 0.34 & \pm & 0.01 & 0.27 & \pm & 0.01 & 1.32 & \pm & 0.01 & 0.21 & \pm & 0.01 & 0.13 & \pm & 0.01 & 0.011 & \pm & 0.01 & 1.45 & \pm & 0.00 & 0.18 & \pm & 0.01 \\
\hline \multirow[t]{2}{*}{ Control } & SNP $0.4 \mathrm{mM}$ & 0.38 & \pm & $0.12^{c}$ & 0.30 & \pm & $0.00^{c}$ & 1.51 & \pm & $0.05^{c}$ & 0.23 & \pm & $0.01^{c}$ & 0.14 & \pm & $0.01^{c}$ & 0.011 & \pm & $0.01^{\mathrm{c}}$ & 1.72 & \pm & $0.01^{c}$ & 0.21 & \pm & $0.05^{\mathrm{c}}$ \\
\hline & SNP $0.6 \mathrm{mM}$ & 0.30 & \pm & $0.01^{\mathrm{c}}$ & 0.20 & \pm & $0.01^{\mathrm{c}}$ & 1.05 & \pm & $0.32^{\mathrm{C}}$ & 0.18 & \pm & $0.01^{\mathrm{c}}$ & 0.10 & \pm & $0.01^{\mathrm{c}}$ & 0.006 & \pm & $0.00^{c}$ & 1.68 & \pm & $0.01^{\mathrm{c}}$ & 0.18 & \pm & $0.01^{c}$ \\
\hline Nickel sulphate & $100 \mathrm{mM}$ & 2.61 & \pm & $0.01^{\mathrm{c}}$ & 0.14 & \pm & $0.01^{\mathrm{c}}$ & 0.70 & \pm & $0.04^{c}$ & 0.14 & \pm & $0.01^{c}$ & 0.06 & \pm & $0.00^{c}$ & 0.006 & \pm & $0.00^{c}$ & 0.60 & \pm & $0.00^{c}$ & 0.10 & \pm & $0.01^{\mathrm{c}}$ \\
\hline \multicolumn{2}{|c|}{$\begin{array}{l}\text { Nickel sulphate } 100 \mathrm{mM} \\
+ \text { SNP0.4mM }\end{array}$} & 0.35 & \pm & $0.01^{* * *}$ & 0.21 & \pm & $0.01^{* * *}$ & 1.05 & \pm & $0.00^{* * *}$ & 0.16 & \pm & $0.01^{* * *}$ & 0.10 & \pm & $0.01^{* * *}$ & 0.009 & \pm & $0.00^{* * *}$ & 1.59 & \pm & $0.01^{* * *}$ & 0.19 & \pm & $0.02^{* * *}$ \\
\hline \multicolumn{2}{|c|}{$\begin{array}{l}\text { Nickel sulphate } 100 \mathrm{mM} \\
+ \text { SNP0.6mM }\end{array}$} & 0.29 & \pm & $0.00^{* * *}$ & 0.18 & \pm & $0.00^{* * *}$ & 0.74 & \pm & $0.01^{* * *}$ & 0.14 & \pm & $0.03^{* * *}$ & 0.09 & \pm & $0.00^{* * *}$ & 0.008 & \pm & $0.00^{* * *}$ & 1.27 & \pm & $0.00^{* * *}$ & 0.16 & \pm & $0.02^{* * *}$ \\
\hline Nickel sulphate & $150 \mathrm{mM}$ & 0.23 & \pm & $0.01^{\mathrm{c}}$ & 0.10 & \pm & $0.01^{\mathrm{c}}$ & 0.49 & \pm & $0.01^{\mathrm{c}}$ & 0.09 & \pm & $0.01^{c}$ & 0.04 & \pm & $0.01^{\mathrm{c}}$ & 0.005 & \pm & $0.01^{c}$ & 0.60 & \pm & $0.00^{c}$ & 0.04 & \pm & $0.01^{\mathrm{c}}$ \\
\hline \multicolumn{2}{|c|}{ Nickel sulphate 150mM } & 0.34 & \pm & $0.01^{* * *}$ & 0.19 & \pm & $0.01^{* * *}$ & 0.90 & \pm & $0.03^{* * *}$ & 0.14 & \pm & $0.01^{* * *}$ & 0.05 & \pm & $0.00^{* * *}$ & 0.009 & \pm & $0.01^{* * *}$ & 1.48 & \pm & $0.01^{* * *}$ & 0.15 & \pm & $0.01^{* * *}$ \\
\hline \multicolumn{2}{|c|}{ 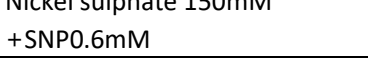 } & 0.27 & \pm & $0.01^{* * *}$ & 0.17 & \pm & $0.01^{* * *}$ & 0.69 & \pm & $0.01^{* * *}$ & 0.09 & \pm & $0.00^{* * *}$ & 0.03 & \pm & $0.00^{* * *}$ & 0.005 & \pm & $0.01^{* * *}$ & 0.79 & \pm & $0.00^{* * *}$ & 0.13 & \pm & $0.03^{* * *}$ \\
\hline
\end{tabular}

Values with a superscript are significantly different from the control (tap water). Letter "a" at $\mathrm{P}<0.05$ (significant), "b" at $\mathrm{P}<0.01$ (highly significant), "c" at $\mathrm{P}<0.001$ (extremely significant), and the absence of letter $=$ non-significant.
Values with a superscript are significantly different from the control (SNP0.4mM, SNP0.6mM). Asterisk ${ }^{*}$ at $\mathrm{P}<0.05$ (significant), ${ }^{* *}$ at $\mathrm{P}<0.01$ (highly significant), ${ }^{* * *}$ at $\mathrm{P}<0.001$ (extremely significant), and the absence of letter $=$ non-significant. 
Table 4. Effect of exogenous application of SNP (as NO donor) on photosynthetic pigments content of Lupinus termis L. leaves under Ni stress (0, 100 and 150 mM). Results are expressed as $\mu \mathrm{g} \mathrm{g}^{-1}$ fresh weight. Data are mean \pm SE (Standard Error) of five replicates.

\begin{tabular}{|c|c|c|c|c|c|c|c|c|c|c|c|c|c|}
\hline \multicolumn{2}{|c|}{ Treatment } & \multicolumn{3}{|c|}{ Chlorophyll a } & \multicolumn{3}{|c|}{ Chlorophyll b } & \multicolumn{3}{|c|}{ Carotenoids } & \multicolumn{3}{|c|}{ Total pigments } \\
\hline \multirow{3}{*}{ Control } & $\mathrm{H}_{2} \mathrm{O}$ & 3.41 & \pm & 0.01 & 1.37 & \pm & 0.05 & 1.93 & \pm & 0.02 & 6.70 & \pm & 0.06 \\
\hline & SNP $0.4 \mathrm{mM}$ & 4.53 & \pm & $0.06^{\mathrm{b}}$ & 2.37 & \pm & $0.10^{\mathrm{b}}$ & 3.49 & \pm & $0.08^{\mathrm{b}}$ & 10.39 & \pm & $0.22^{\mathrm{b}}$ \\
\hline & SNP $0.6 \mathrm{mM}$ & 3.98 & \pm & $0.04^{\mathrm{b}}$ & 2.16 & \pm & 0.21 & 3.16 & \pm & $0.09^{\mathrm{b}}$ & 9.29 & \pm & $0.28^{\mathrm{b}}$ \\
\hline Nickel sulphate & $100 \mathrm{mM}$ & 0.86 & \pm & $0.00^{\mathrm{C}}$ & 0.69 & \pm & $0.01^{b}$ & 0.41 & \pm & $0.03^{c}$ & 1.96 & \pm & $0.03^{c}$ \\
\hline \multicolumn{2}{|c|}{ Nickel sulphate $100 \mathrm{mM}+\mathrm{SNP} 0.4 \mathrm{mM}$} & 1.98 & \pm & $0.34^{*}$ & 1.46 & \pm & $0.04^{* *}$ & 1.49 & \pm & $0.13^{* * *}$ & 4.93 & \pm & $0.47^{* *}$ \\
\hline \multicolumn{2}{|c|}{ Nickel sulphate $100 \mathrm{mM}+\mathrm{SNP} 0.6 \mathrm{mM}$} & 0.96 & \pm & $0.00^{* * *}$ & 0.69 & \pm & ${ }^{*} 0.01$ & 0.38 & \pm & $0.00^{* * *}$ & 2.03 & \pm & $0.01^{* * *}$ \\
\hline Nickel sulphate & $150 \mathrm{mM}$ & 0.70 & \pm & $0.01^{\mathrm{c}}$ & 0.52 & \pm & $0.00^{\mathrm{b}}$ & 0.40 & \pm & $0.01^{\mathrm{c}}$ & 1.62 & \pm & $0.01^{c}$ \\
\hline \multicolumn{2}{|c|}{ Nickel sulphate $150 \mathrm{mM}+\mathrm{SNP} 0.4 \mathrm{mM}$} & 1.00 & \pm & $0.00^{* * *}$ & 0.79 & \pm & $0.01^{* *}$ & 0.88 & \pm & $0.10^{* * *}$ & 2.67 & \pm & $0.09^{* * *}$ \\
\hline \multicolumn{2}{|c|}{ Nickel sulphate $150 \mathrm{mM}+\mathrm{SNP} 0.6 \mathrm{mM}$} & 0.35 & \pm & $0.00^{* * *}$ & 0.28 & \pm & $0.00^{*}$ & 0.21 & \pm & $0.01^{* * *}$ & 0.84 & \pm & $0.01^{* * *}$ \\
\hline
\end{tabular}

Values with a superscript are significantly different from the control (tap water). Letter "a" at $\mathrm{P}<0.05$ (significant), "b" at $\mathrm{P}<0.01$ (highly significant), "c" at $\mathrm{P}<0.001$ (extremely significant), and the absence of letter $=$ non-significant. Values with a superscript are significantly different from the control (SNP0.4mM, SNP0.6mM). Asterisk * at P<0.05 (significant), $* *$ at $P<0.01$ (highly significant), ${ }^{* * *}$ at $\mathrm{P}<0.001$ (extremely significant), and the absence of letter $=$ non-significant.

Table 5. Effect of exogenous application of SNP (as NO donor) on soluble sugar, polysaccharide and total carbohydrates content of Lupinus termis L. under N

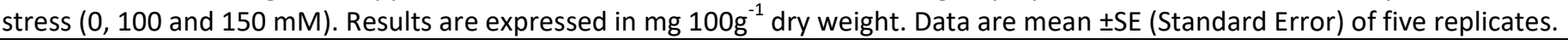

\begin{tabular}{|c|c|c|c|c|c|c|c|c|c|c|c|c|c|c|c|c|c|c|c|}
\hline \multirow{2}{*}{\multicolumn{2}{|c|}{ Treatment }} & \multicolumn{6}{|c|}{ Soluble Sugar } & \multicolumn{6}{|c|}{ Polysaccharide } & \multicolumn{6}{|c|}{ Total carbohydrates } \\
\hline & & \multicolumn{3}{|c|}{ Shoot } & \multicolumn{3}{|c|}{ Root } & \multicolumn{3}{|c|}{ Shoot } & \multicolumn{3}{|c|}{ Root } & \multicolumn{3}{|c|}{ Shoot } & \multicolumn{3}{|c|}{ Root } \\
\hline \multirow{3}{*}{ Control } & $\mathrm{H}_{2} \mathrm{O}$ & 894.44 & \pm & 11.56 & 1035.18 & \pm & 1.85 & 2899.63 & \pm & 1.77 & 2253.70 & \pm & 12.96 & 3794.07 & \pm & 13.30 & 3290.74 & \pm & 14.46 \\
\hline & SNP $0.4 \mathrm{mM}$ & 1148.15 & \pm & $9.80^{\mathrm{C}}$ & 1092.59 & \pm & 18.51 & 3504.81 & \pm & $28.26^{\mathrm{b}}$ & 2401.67 & \pm & $3.69^{\mathrm{b}}$ & 4652.97 & \pm & $28.98^{\mathrm{C}}$ & 3506.42 & \pm & $7.11^{\mathrm{c}}$ \\
\hline & SNP $0.6 \mathrm{mM}$ & 794.45 & \pm & $5.55^{\mathrm{b}}$ & 855.55 & \pm & $38.88^{a}$ & 3016.67 & \pm & $5.56^{\mathrm{c}}$ & 1935.18 & \pm & $1.85^{\mathrm{c}}$ & 3811.11 & \pm & 5.55 & 2778.39 & \pm & $35.82^{\mathrm{b}}$ \\
\hline Nickel sulphate & $100 \mathrm{mM}$ & 635.18 & \pm & $1.85^{\mathrm{b}}$ & 588.89 & \pm & $5.55^{c}$ & 1633.33 & \pm & $202.78^{\mathrm{a}}$ & 1755.56 & \pm & $0.96^{c}$ & 2268.52 & \pm & $201.88^{\mathrm{a}}$ & 2343.15 & \pm & $4.38^{c}$ \\
\hline \multicolumn{2}{|c|}{$\begin{array}{l}\text { Nickel sulphate } 100 \mathrm{mM} \\
+ \text { SNP0.4mM }\end{array}$} & 801.85 & \pm & $1.85^{* * *}$ & 1033.33 & \pm & 0.00 & 2512.96 & \pm & $4.89^{* * *}$ & 1926.18 & \pm & $9.13^{* * *}$ & 3314.81 & \pm & $3.70^{* * *}$ & 2953.43 & \pm & $3.05^{* * *}$ \\
\hline \multicolumn{2}{|c|}{ Nickel sulphate $100 \mathrm{mM}$} & 377.78 & \pm & $39.02^{* *}$ & 968.52 & \pm & 1.85 & 2392.59 & \pm & $3.70^{* * *}$ & 1616.67 & \pm & $5.55^{* * *}$ & 2770.37 & \pm & $40.74^{* * *}$ & 2585.80 & \pm & $4.82^{*}$ \\
\hline Nickel sulphate & $150 \mathrm{mM}$ & 229.63 & \pm & $18.52^{c}$ & 568.52 & \pm & $12.96^{\mathrm{c}}$ & 1485.74 & \pm & $4.42^{\mathrm{c}}$ & 1428.15 & \pm & $0.18^{\mathrm{c}}$ & 1715.37 & \pm & $22.88^{\mathrm{c}}$ & 1988.15 & \pm & $4.26^{c}$ \\
\hline \multicolumn{2}{|c|}{ Nickel sulphate $150 \mathrm{mM}$} & 416.66 & \pm & $16.03^{* * *}$ & 590.74 & \pm & $1.85^{* * *}$ & 1582.03 & \pm & $1.29^{* * *}$ & 1465.93 & \pm & $0.18^{* * *}$ & 1998.70 & \pm & $14.92^{* * *}$ & 2057.22 & \pm & $1.47^{* * *}$ \\
\hline \multicolumn{2}{|c|}{$\begin{array}{l}\text { Nickel sulphate } 150 \mathrm{mM} \\
+ \text { SNP0.6mM }\end{array}$} & 244.44 & \pm & $0.00^{* * *}$ & 246.29 & \pm & $1.85^{* *}$ & 1419.44 & \pm & $0.32^{* * *}$ & 1279.63 & \pm & $0.37^{* * *}$ & 1663.89 & \pm & $0.32^{* * *}$ & 1524.94 & \pm & $0.49^{* * *}$ \\
\hline
\end{tabular}

Values with a superscript are significantly different from the control (tap water). Letter "a" at $\mathrm{P}<0.05$ (significant), "b" at $\mathrm{P}<0.01$ (highly significant), "c" at $\mathrm{P}<0.001$ (extremely significant), and the absence of letter $=$ non-significant. non-significant 
Table 6.Effect of exogenous application of SNP (as NO donor) on proline content of Lupinus termis L. under Ni stress (0, 100 and $150 \mathrm{mM})$. Results are

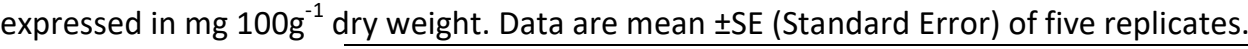

\begin{tabular}{|c|c|c|c|c|c|c|c|}
\hline \multicolumn{2}{|l|}{ Treatment } & \multicolumn{3}{|c|}{ Shoot } & \multicolumn{3}{|c|}{ Root } \\
\hline Control & $\mathrm{H}_{2} \mathrm{O}$ & 2.29 & \pm & 0.19 & 1.05 & \pm & 0.10 \\
\hline SNP & $0.4 \mathrm{mM}$ & 2.15 & \pm & 0.01 & 0.76 & \pm & 0.00 \\
\hline SNP & $0.6 \mathrm{mM}$ & 2.27 & \pm & 0.05 & 0.90 & \pm & 0.07 \\
\hline Nickel sulphate & $100 \mathrm{mM}$ & 3.19 & \pm & $0.07^{\mathrm{a}}$ & 1.12 & \pm & 0.00 \\
\hline Nickel sulphate $100 \mathrm{mM}+\& \mathrm{SNP} 0.4 \mathrm{mM}$ & & 2.58 & \pm & 0.22 & 1.01 & \pm & $0.01^{* * *}$ \\
\hline Nickel sulphate $100 \mathrm{mM}+\mathrm{sNP} 0.6 \mathrm{mM}$ & & 2.66 & \pm & 0.14 & 1.02 & \pm & 0.01 \\
\hline Nickel sulphate & $150 \mathrm{mM}$ & $\begin{array}{l}3.95 \\
2.85\end{array}$ & $\begin{array}{l} \pm \\
\pm\end{array}$ & $\begin{array}{l}1.24 \\
0.04^{* *}\end{array}$ & $\begin{array}{l}1.53 \\
1.09\end{array}$ & $\begin{array}{l} \pm \\
\pm\end{array}$ & $\begin{array}{l}0.05^{\mathrm{a}} \\
0.05^{*}\end{array}$ \\
\hline Nickel sulphate $150 \mathrm{mM}+\mathrm{sNP} 0.4 \mathrm{mM}$ & & & & & & & \\
\hline & & 3.29 & \pm & $0.08^{* * *}$ & 1.20 & \pm & $0.00^{*}$ \\
\hline
\end{tabular}

Values with a superscript are significantly different from the control (tap water). Letter "a" at $\mathrm{P}<0.05$ (significant), "b" at $\mathrm{P}<0.01$ (highly significant), "c" at $\mathrm{P}<0.001$ (extremely significant), and the absence of letter $=$ non-significant. Values with a superscript are significantly different from the control (SNP0.4mM, SNP0.6mM). Asterisk ${ }^{*}$ at $\mathrm{P}<0.05$ (significant), ${ }^{* *}$ at $\mathrm{P}<0.01$ (highly significant), ${ }^{* * *}$ at $\mathrm{P}<0.001$ (extremely significant), and the absence of letter $=$ non-significant.

Table 7. Effect of exogenous application of SNP (as NO donor) on mineral ions (Ca ${ }^{2+}$ and $\mathrm{Mg}^{2+}$ content) and $\mathrm{Ni}^{2+}$ content of Lupinus termis $\mathrm{L}$. under $\mathrm{Ni}$ stress ( 0 , 100 and 150 $\mathrm{mM}$ ). Results are expressed in $\mathrm{mg} \mathrm{g}^{-1}$ dry weight. Data are mean \pm SE (Standard Error) of five replicates.

\begin{tabular}{|c|c|c|c|c|c|c|c|c|c|c|c|c|c|c|c|c|c|c|c|}
\hline \multirow{2}{*}{ Treatment } & \multirow[t]{2}{*}{ Mineral Ions } & \multirow[t]{2}{*}{$\mathrm{Ca}^{2+}$} & & & & & & \multicolumn{6}{|l|}{$\mathrm{Mg}^{2+}$} & \multicolumn{6}{|l|}{$\mathrm{Ni}^{2+}$} \\
\hline & & & \multicolumn{3}{|c|}{ Shoot } & \multicolumn{2}{|c|}{ Root } & \multicolumn{3}{|c|}{ Shoot } & \multicolumn{3}{|c|}{ Root } & \multicolumn{3}{|c|}{ Shoot } & \multicolumn{3}{|c|}{ Root } \\
\hline \multirow{3}{*}{ Control } & $\mathrm{H}_{2} \mathrm{O}$ & 2.90 & \pm & 0.06 & $\begin{array}{l}2.9 \\
3\end{array}$ & \pm & 0.06 & 14.02 & \pm & 0.04 & 12.27 & \pm & 0.18 & 27.97 & \pm & 0.32 & 38.27 & \pm & 0.38 \\
\hline & SNP $0.4 \mathrm{mM}$ & 3.54 & \pm & $0.03^{b}$ & $\begin{array}{l}3.3 \\
1\end{array}$ & \pm & 0.21 & 14.60 & \pm & $0.06^{b}$ & 17.51 & \pm & $0.06^{c}$ & 7.69 & \pm & $0.25^{c}$ & 26.33 & \pm & $0.88^{b}$ \\
\hline & SNP $0.6 \mathrm{mM}$ & 2.94 & \pm & 0.03 & $\begin{array}{l}3.1 \\
7\end{array}$ & \pm & $0.04^{b}$ & 14.03 & \pm & 0.04 & 12.21 & \pm & 0.01 & 48.10 & \pm & $1.06^{c}$ & 67.01 & \pm & $1.72^{\mathrm{b}}$ \\
\hline Nickel sulphate & $100 \mathrm{mM}$ & 6.60 & \pm & $0.06^{c}$ & $\begin{array}{l}6.3 \\
3\end{array}$ & \pm & $0.17^{b}$ & 3.62 & \pm & $0.40^{c}$ & 9.03 & \pm & $0.02^{b}$ & 350.08 & \pm & $1.09^{c}$ & 367.20 & \pm & $1.56^{\mathrm{c}}$ \\
\hline \multicolumn{2}{|c|}{$\begin{array}{l}\text { Nickel sulphate } \\
100 \mathrm{mM}+\text { SNPO.4mM }\end{array}$} & 7.15 & \pm & $0.08^{* * *}$ & $\begin{array}{l}7.0 \\
3\end{array}$ & \pm & $0.03^{* *}$ & 12.50 & \pm & $0.06^{* * *}$ & 10.70 & \pm & $0.01^{* * *}$ & 140.05 & \pm & $2.27^{* * *}$ & 215.50 & \pm & $2.47^{* * *}$ \\
\hline \multicolumn{2}{|c|}{$\begin{array}{l}\text { Nickel sulphate } 100 \mathrm{mM} \\
+ \text { +SNP0.6mM }\end{array}$} & 6.17 & \pm & $0.17^{* *}$ & $\begin{array}{l}6.1 \\
7\end{array}$ & \pm & $0.17^{* *}$ & 10.39 & \pm & $0.01^{* * *}$ & 5.56 & \pm & $0.02^{* * *}$ & 368.09 & \pm & $1.07^{* * *}$ & 319.38 & \pm & $1.72^{* * *}$ \\
\hline Nickel sulphate & $150 \mathrm{mM}$ & 4.63 & \pm & $0.09^{c}$ & $\begin{array}{l}4.5 \\
0\end{array}$ & \pm & $0.00^{c}$ & 4.12 & \pm & $0.04^{\mathrm{c}}$ & 1.90 & \pm & $0.06^{\mathrm{c}}$ & 690.32 & \pm & $10.69^{c}$ & 1254.92 & \pm & $0.65^{c}$ \\
\hline \multirow{2}{*}{\multicolumn{2}{|c|}{$\begin{array}{l}\text { Nickel sulphate } 150 \mathrm{mM} \\
+ \text { +SNPO.4mM } \\
\text { Nickel sulphate } 150 \mathrm{mM} \\
+ \text { +SNP0.6mM }\end{array}$}} & 6.20 & \pm & $0.11^{* * *}$ & $\begin{array}{l}6.0 \\
3\end{array}$ & \pm & $0.03^{* *}$ & 8.19 & \pm & $0.01^{* * *}$ & 4.19 & \pm & $0.00^{* * *}$ & 249.85 & \pm & $0.71^{* * *}$ & 377.42 & \pm & $1.39^{* * *}$ \\
\hline & & 5.37 & \pm & $0.18^{* *}$ & $\begin{array}{l}5.5 \\
7\end{array}$ & \pm & $0.07^{* * *}$ & 4.05 & \pm & $0.01^{* * *}$ & 4.16 & \pm & $0.01^{* * *}$ & 633.99 & \pm & $0.58^{* * *}$ & 690.09 & \pm & $10.93^{*}$ \\
\hline
\end{tabular}

+ SNP0.6mM

Values with a superscript are significantly different from the control (tap water). Letter "a" at $\mathrm{P}<0.05$ (significant), "b" at $\mathrm{P}<0.01$ (highly significant), "c" at $\mathrm{P}<0.001$ (extremely significant), and the absence of letter $=$ non-significant. non-significant. 
Table 8. Effect of exogenous application of SNP (as NO donor) on some oxidative markers (Hydrogen Peroxide, electrolyte leakage and lipid peroxidation content) of Lupinus termis L. under Ni stress (0, 100 and $150 \mathrm{mM}$ ). Data are mean \pm SE (Standard Error) of five replicates.

\begin{tabular}{|c|c|c|c|c|c|c|c|c|c|c|c|c|c|c|c|c|}
\hline \multirow[t]{3}{*}{ Treatment } & \multirow[b]{3}{*}{$\mathrm{H}_{2} \mathrm{O}$} & \multicolumn{6}{|c|}{ Hydrogen Peroxide $\left(\mathrm{mM} \mathrm{g}^{-1}\right)$} & \multicolumn{3}{|c|}{ Electrolyte Leakage (\%) } & \multicolumn{6}{|c|}{$\begin{array}{l}\text { Lipid Peroxidation (MDA) } \\
\left(\mathrm{mM} \mathrm{g}^{-1}\right)\end{array}$} \\
\hline & & \multicolumn{3}{|c|}{ Shoot } & \multicolumn{3}{|c|}{ Root } & \multicolumn{3}{|c|}{ Shoot } & \multicolumn{3}{|c|}{ Shoot } & \multicolumn{3}{|c|}{ Root } \\
\hline & & 16.92 & \pm & 0.23 & 1.47 & \pm & 0.01 & 19.29 & \pm & 0.00 & 0.00059 & \pm & 0.0000 & 0.00038 & \pm & 0.0000 \\
\hline \multirow[t]{2}{*}{ Control } & SNP $0.4 \mathrm{mM}$ & 14.02 & \pm & $0.43^{\mathrm{a}}$ & 1.04 & \pm & $0.02^{\mathrm{c}}$ & 15.39 & \pm & $0.00^{c}$ & 0.00034 & \pm & $0.0000^{c}$ & 0.00022 & \pm & $0.0000^{\mathrm{b}}$ \\
\hline & SNP $0.6 \mathrm{mM}$ & 18.12 & \pm & $0.32^{\mathrm{a}}$ & 1.22 & \pm & $0.01^{\mathrm{c}}$ & 19.02 & \pm & 0.17 & 0.00047 & \pm & $0.0000^{c}$ & 0.00028 & \pm & $0.0000^{\mathrm{a}}$ \\
\hline Nickel sulphate & $100 \mathrm{mM}$ & 55.24 & \pm & $0.17^{c}$ & 17.74 & \pm & $0.13^{c}$ & 61.88 & \pm & $0.00^{c}$ & 0.00132 & \pm & $0.0000^{c}$ & 0.00046 & \pm & $0.0000^{b}$ \\
\hline \multicolumn{2}{|c|}{ Nickel sulphate $100 \mathrm{mM}+\mathrm{SNPO} .4 \mathrm{mM}$} & 38.32 & \pm & $0.13^{* * *}$ & 6.44 & \pm & $0.11^{* * *}$ & 21.64 & \pm & 3.32 & 0.00064 & \pm & 0.0000 & 0.00022 & \pm & 0.0000 \\
\hline \multicolumn{2}{|c|}{ Nickel sulphate $100 \mathrm{mM}+\mathrm{SNP0.6 \textrm {mM }}$} & 51.27 & \pm & $0.06^{* * *}$ & 8.59 & \pm & $0.73^{*}$ & 27.89 & \pm & $0.00^{* * *}$ & 0.00070 & \pm & 0.0001 & 0.00024 & \pm & 0.0000 \\
\hline Nickel sulphate & $150 \mathrm{mM}$ & 82.89 & \pm & $0.50^{c}$ & 19.44 & \pm & $1.12^{\mathrm{b}}$ & 74.49 & \pm & $0.00^{\mathrm{c}}$ & 0.00196 & \pm & $0.0000^{c}$ & 0.00056 & \pm & 0.0001 \\
\hline \multicolumn{2}{|c|}{ Nickel sulphate $150 \mathrm{mM}+\mathrm{SNP} 0.4 \mathrm{mM}$} & 40.97 & \pm & $0.92^{* * *}$ & 7.39 & \pm & $0.11^{* * *}$ & 28.21 & \pm & $0.00^{* * *}$ & 0.00068 & \pm & $0.0000^{* * *}$ & 0.00032 & \pm & $0.0000^{* *}$ \\
\hline \multicolumn{2}{|c|}{ Nickel sulphate $150 \mathrm{mM}+S N P 0.6 \mathrm{mM}$} & 80.81 & \pm & $0.06^{* * *}$ & 9.79 & \pm & $0.17^{* * *}$ & 31.61 & \pm & $0.00^{* * *}$ & 0.00075 & \pm & 0.0000 & 0.00036 & \pm & $0.0000^{*}$ \\
\hline
\end{tabular}

Values with a superscript are significantly different from the control (tap water). Letter "a" at $\mathrm{P}<0.05$ (significant), "b" at $\mathrm{P}<0.01$ (highly significant), "c" at $\mathrm{P}<0.001$ (extremely significant), and the absence of letter $=$ non-significant.
Values with a superscript are significantly different from the control (SNP0.4mM, SNP0.6mM). Asterisk * at $\mathrm{P}<0.05$ (significant), $* *$ at $\mathrm{P}<0.01$ (highly significant), *** at $\mathrm{P}<0.001$ (extremely significant), and the absence of letter $=$ non-significant. 


\section{Carbohydrate contents}

The effects of SNP treatments on the carbohydrate (soluble sugars, polysaccharides and total carbohydrates) contents of lupine shoots and roots were concentration-dependent (Table 5). The lower SNP concentration $(0.4 \mathrm{mM})$ had a stimulatory effect, while the higher concentration $(0.6 \mathrm{mM})$ had an inhibitory effect, when compared with water-treated control plants. The stimulatory effect of the 0.4-mM SNP treatment was much more pronounced in shoots than roots and was evaluated as $28.37 \%, 20.87 \%$ and $22.64 \%$ increases in soluble sugars, polysaccharides and total carbohydrates, respectively, in 0.4-mM SNP-treated shoots over the control values.

Treating Ni-stressed plants with $0.4 \mathrm{mM}$ SNP partially alleviated the inhibitory effect of $\mathrm{Ni}$ toxicity on the soluble sugar content of lupine and increased the amounts of polysaccharides accumulated in both shoots and roots. Meanwhile, treating with Ni sulfate together with $0.6 \mathrm{mM} \mathrm{SNP}$ resulted in a continuous decrease in the soluble sugar contents of shoots to values lower than those of plants treated with 0.6 $\mathrm{mM}$ SNP alone. The extremely significant decrease was evaluated as $52.45 \%$ and $69.23 \%$ in plants treated with $0.6 \mathrm{mM}$ SNP and subjected to 100 and $150 \mathrm{mM} \mathrm{Ni}$ sulfate stress, respectively, compared with the corresponding control plants treated with $0.6 \mathrm{mM}$ SNP alone.

The maximum total carbohydrate content in lupine shoots in treated plants followed the order of: $0.4 \mathrm{mM}$ SNP > control plants (tap water) $>100 \mathrm{mM} \mathrm{Ni}$ sulfate $+0.4 \mathrm{mM} \mathrm{SNP}>0.6$ $\mathrm{mM}$ SNP > $150 \mathrm{mM} \mathrm{Ni}+0.6 \mathrm{mM}$ SNP.

\section{Proline content}

The changes in proline content in response to treatment with SNP and/or Ni sulfate are illustrated in Table 6. The SNP treatment decreased the proline content, while $\mathrm{Ni}$ sulfate applications increased it in both shoots and roots of lupine plants. The maximum proline accumulation was detected in response to $150 \mathrm{mM} \mathrm{Ni}$ sulfate and was evaluated as $72.49 \%$ greater in shoots compared with unstressed control plants. The lowest proline value was detected in plants treated with $0.4 \mathrm{mM}$ SNP and evaluated as $27.62 \%$ less in roots compared with the untreated control. Plants treated with both SNP and $\mathrm{Ni}$ sulfate recorded intermediate proline content, greater than those treated with SNP alone and less than those exposed to Ni sulfate alone.

\section{Organic acids profile}

The data in Figure 1 represent the changes in organic acid profiles in shoots and roots of $L$. termis $L$. in response to treatments with SNP and/or Ni sulfate. Generally, the shoot organic acid contents were much greater than in roots. Among the organic acids, butyric and malic acids were the most abundant compounds in shoots, while citric and malic acids were the major compounds in roots. Applications of SNP and/or Ni sulfate caused marked changes in the organic acid profiles of lupine plants, compared with the control. Exogenous applications of NO (in the form of SNP at 0.4 and $0.6 \mathrm{mM}$ ) significantly increased citric, malic, succinic, formic, acetic, fumaric and butyric acid levels. The increase was much more pronounced in plants treated with $0.4 \mathrm{mM}$ SNP and was evaluated as $73.78 \%, 13.09 \%, 249.12 \%, 173.53 \%, 31.24 \%$, $85.714 \%$ and $114.80 \%$ in shoots, and $19.713 \%, 266.8 \%$, $453.4 \%, 86.206 \%, 34.75 \%, 233.00 \%$ and $633.3 \%$ in roots, respectively, over the untreated control values. However, oxalic, maleic and propionic acids were increased only in response to the low concentration of SNP $(0.4 \mathrm{mM})$ and significantly decreased in response to the higher concentration (0.6 mM SNP).

$\mathrm{Ni}$ toxicity had a significant effect on the organic acid composition of lupine plants. Oxalic, citric, maleic and acetic acids were increased in both shoots and roots in response to both applied concentrations of Ni sulfate, while malic, succinic and propionic acids were increased only in roots and decreased in shoots compared with the control. In most cases, Fumaric and formic acids were significantly inhibited in both shoots and roots in response to $\mathrm{Ni}$ treatments. As a percentage, acetic acid was most affected by $\mathrm{Ni}$ toxicity. The accumulated acetic acid increased by $274.38 \%$ and $64.79 \%$ in shoots and $31.03 \%$ and $175.64 \%$ in roots in response to 100 and $150-\mathrm{mM}$ Ni sulfate treatments, respectively.

Treating lupine plants with a combination of Ni sulfate and SNP significantly increased the accumulated amounts of oxalic, citric and maleic acids over those treated with SNP or Ni alone. Maximum values were detected in response to $150 \mathrm{mM} \mathrm{Ni}+$ $0.4 \mathrm{mM}$ SNP. NO production (using 0.4 and $0.6 \mathrm{mM}$ SNP) completely overcame the inhibitory effect of $\mathrm{Ni}$ on the malic acid content, and the maximum values were detected in plants treated with $100 \mathrm{mM} \mathrm{Ni}$ sulfate + $0.4 \mathrm{mM} \mathrm{SNP}$, at $151.70 \%$ and $23.25 \%$ in shoots and roots, respectively, over $0.4 \mathrm{mM}$ SNP as the control value. However, only the $0.4-\mathrm{mM}$ SNP treatment could alleviate the inhibitory effect of $\mathrm{Ni}$ toxicity on the production of succinate and butyric acid.

A highly significant increase in formic acid and acetic acid contents was occurred, when different concentrations of $\mathrm{Ni}$ sulfate together with SNP applied. The highest values in shoot were detected by applying $100 \mathrm{mM}$ Ni sulfate $+0.4 \mathrm{mM}$ SNP (8.69- and 5.54-fold in case of formic acid and acetic acid, respectively), whereas the maximum values in root were detected in response to $150 \mathrm{mM} \mathrm{Ni}+0.4 \mathrm{mM}$ SNP (9.43 and 3.79-fold in formic acid and acetic acid, respectively) of untreated control value.

All of the treatments caused an increase in the propionic acid content of roots of Lupinus termis L., when different concentrations of $\mathrm{Ni}$ sulfate were applied together with 0.4 $\mathrm{mM}$ SNP, compared with the $0.4 \mathrm{mM}$ SNP control value. The highest extremely significant increase $(P<0.001)$ was detected when $150 \mathrm{mM}$ Ni sulfate $+0.4 \mathrm{mM}$ SNP was applied and was calculated as $13.79 \%$ greater than the $0.4 \mathrm{mM}$ SNP control value.

\section{Mineral ion contents and $\mathrm{Ni}^{2+}$ accumulation}

The effects of SNP treatments and Ni toxicity on the $\mathrm{Mg}^{2+}$ and $\mathrm{Ca}^{2+}$ contents and $\mathrm{Ni}$ accumulation in lupine plants are presented in Table 7.

The effect of SNP on divalent cations is concentration dependent. The 0.4-mM SNP treatment highly significantly increased the $\mathrm{Mg}^{2+}$ and $\mathrm{Ca}^{2+}$ concentrations compared with the 
untreated control, while the $0.6-\mathrm{mM}$ SNP treatment had no significant effects on their contents.

$\mathrm{Ni}$ toxicity caused extremely significant increases in $\mathrm{Ca}^{2+}$ and highly significant decreases in $\mathrm{Mg}^{2+}$ contents in both shoots and roots as compared with the untreated control. The reductions in $\mathrm{Mg}^{2+}$ were calculated as $74.18 \%$ and $70.61 \%$ in shoots and $26.41 \%$ and $84.52 \%$ in roots of plants treated with 100 and $150 \mathrm{mM}$ Ni sulfate, respectively. The treatment of $\mathrm{Ni}$ stressed plants with $0.4 \mathrm{mM}$ SNP partially alleviated the inhibitory effect of $\mathrm{Ni}$ on the $\mathrm{Mg}^{2+}$ level and led to the accumulation of additional amounts of $\mathrm{Ca}^{2+}$.

The endogenous $\mathrm{Ni}^{2+}$ ion content showed extremely significant increases $(P<0.001)$ in both shoots and roots of $L$. termis $L$. in response to $\mathrm{Ni}$ sulfate treatments, as compared with the untreated control. The detected increase was much greater in roots than shoots and was directly proportional to the concentration of $\mathrm{Ni}$ applied. The highest accumulation of $\mathrm{Ni}$ ions was found in L. termis plants receiving $150 \mathrm{mM}$ Ni sulfate and was evaluated as 24.68- and 32.76-fold greater than the control values in shoots and roots, respectively. The treatment of Ni-stressed plants with 0.4 and $0.6 \mathrm{mM} \mathrm{SNP}$ extremely and significantly reduced the amounts of $\mathrm{Ni}$ accumulated in the plants. The lower dose of SNP $(0.4 \mathrm{mM})$ was more effective in reducing $\mathrm{Ni}$ accumulation than the higher dose $(0.6 \mathrm{mM})$. The $\mathrm{Ni}$ levels were reduced by $63.81 \%$ and $69.92 \%$ in the shoots and roots of plants, respectively, treated with $150 \mathrm{mM} \mathrm{Ni}$ sulfate + $0.4 \mathrm{mM}$ SNP compared with those treated with 150 $\mathrm{mM}$ Ni sulfate alone.

\section{Oxidative markers}

Oxidative markers, including $\mathrm{H}_{2} \mathrm{O}_{2}$, electrolyte leakage and lipid peroxidation, increased in response to heavy-metal stress with $\mathrm{Ni}$ sulfate (100 mM and $150 \mathrm{mM}$ ) as shown in Table 8, while treatments with both Ni sulfate (100 mM and $150 \mathrm{mM})+\mathrm{SNP}$ (0.4 mM), as a stress inhibitor, decreased $\mathrm{H}_{2} \mathrm{O}_{2}$, electrolyte leakage and lipid peroxidation when compared to Ni-stress treatments alone. The lower SNP concentration $(0.4 \mathrm{mM})$ was more effective than the higher concentration $(0.6 \mathrm{mM})$ in alleviating the oxidative stress caused by different $\mathrm{Ni}$ sulfate concentrations.

\section{Discussion}

The current results indicated that exogenous applications of SNP $(0.4$ and $0.6 \mathrm{mM})$ caused a significant increase in the endogenous NO content in both shoots and roots of lupine compared with the non-treated control. Grossi and D'Angelo (2005) hypothesized that the release mechanism of the NO molecule from SNP involves the sulfhydryl-containing compounds glutathione and cysteine, which help to form the corresponding disulfides and S-nitrosothiols, NO and cyanide ions.

Several past studies on NO functions in plant responses to heavy metals were based on the use of an exogenous NO donor (Hsu and Kao, 2004; Gallego et al., 2005; Laspina et al., 2005). However, stress-induced NO might be endogenously produced and; therefore, play specific roles in responses to heavy metals (Besson-Bard et al., 2009). According to the present results, $\mathrm{Ni}$ sulfate treatments (100 and $150 \mathrm{mM}$ ) caused significant increases in the NO content, and the increases were directly related to the $\mathrm{Ni}$ concentration applied. NO can modulate the expression of metal transporters, such as iron regulated transporter 1 and; therefore, affect the accumulation of these metals in plants (Parani et al., 2004; Besson-Bard et al., 2009). An increase in the endogenous NO content upon exposure to heavy metal (Cd) has also been reported for different crop plants (Groppa et al., 2008; Xiong et al., 2009; Valentovičová et al., 2010). Treatments with $\mathrm{Ni}+$ SNP had an accumulating NO effect in lupine plants. The highest endogenous NO content was detected in plants treated with $0.6 \mathrm{mM} \mathrm{SNP}+150 \mathrm{mM}$ Ni sulfate.

Plant growth is highly influenced by NO. Here, the effect of NO on lupine growth was concentration-dependent, with $0.4 \mathrm{mM}$ SNP causing a significant increase and $0.6 \mathrm{mM}$ SNP causing a significant decrease in all of the measured growth parameters (Table 2). A similar dual behavior of the NO donor, SNP, was also noted by several authors. For example, treating wheat seedlings with a lower concentration of SNP promoted root growth, while a higher concentration was inhibitory (Tian and Lei, 2006). Seedlings of canola, raised from the seeds treated with a lower concentration of SNP had longer roots and greater dry masses, while those treated with a higher concentration had reduced values for these parameters (Zanardo et al., 2005).

The most common response of plants to stress conditions, such as exposure to heavy metals, is growth reduction. A marked decrease was detected in all of the growth parameters in response to 100 and $150 \mathrm{mM} \mathrm{Ni}$ compared with unstressed control plants. Mihailovic and Drazic (2011) illustrated that Ni induced a significant inhibition of leaf and root growth in bean plants after a 4-d exposure, which confirmed previous results (Gajewska and Sklodowska 2005). Interestingly, NO alleviates this effect, similar to the findings of Kopyra and Gwóźdź (2003) and Laspina et al. (2005) that were obtained for lead- and cadmium (Cd)-stressed plants, respectively.

Changes in NO levels are triggered by exogenous plant hormones (Freschi, 2013). In the current study, we detected the changes in endogenous hormones in response to exogenous applications of NO. Endogenous phytohormones, including $I A A, G A_{3}, A B A$ and $J A$, were significantly increased in response to the $0.4 \mathrm{mM}$ SNP treatment. NO appeared to modulate elements controlling either the plant hormone levels (e.g., biosynthesis, degradation and conjugation enzymes), distribution (e.g., transport proteins) or signaling (e.g., receptors and signal transduction proteins). This modulation occurs either at the transcriptional (Xu et al., 2010; Leon and Lozano-Juste, 2011) or post-translational level (Terrile et al., 2012; Feng et al., 2013).

In contrast, all of the applied $\mathrm{Ni}$ sulfate concentrations significantly decreased the hormonal contents in both shoots and roots of lupine plants. Heavy-metal stress leads to a decrease in endogenous levels of auxins. For example, arsenic is able to alter levels of three auxins (IAA, naphthalene-1-acetic acid and indole-3-butyric acid) in Brassica juncea (Srivastava et al. 2013). In another case, a short-term Cd treatment disturbed IAA homeostasis in barley root tips (Zelinova et al., 2015). Besson-Brad et al. (2009) also indicated that Cd suppresses primary root elongation in Arabidopsis. In contrast to our results, increases in the $A B A$ content have been detected in 
germinating chickpea (Cicer arietinum) seeds under lead toxicity conditions (Atici et al., 2005) and in crowberries (Empetrum nigrum) exposed to copper (Cu) and $\mathrm{Ni}$ (Monni et al. 2001). Further studies are needed to reveal the changes in the ABA biosynthesis level caused by applied $\mathrm{Ni}$.

The detected increases in growth-stimulating hormones (such as IAA and $\mathrm{GA}_{3}$ ) could explain the observed growth stimulation in response to NO treatments. The increase in the ABA level in response to NO could explain the protective effect of NO against $\mathrm{Ni}$ toxicity that is connected with $\mathrm{ABA}$-induced stomatal closure. Preventing a decline in the water potential by closing stomata contributes to the plant's adaptability to unfavorable conditions (Pantin et al., 2013). Exposure to toxic metal concentrations impairs the plant's water balance (Mukhopadhyay and Mondal, 2015). Thus, SNP responds to Ni stress by elevating internal hormones.

The two applied SNP concentrations had positive impacts on photosynthetic pigments in lupine leaves. NO enhanced the chlorophyll content in potato, lettuce and Arabidopsis (Beligni and Lamattina, 2000). Additionally, SNP treatments delay yellowing and retard chlorophyll degradation in broccoli (Hyang et al., 2009). They also improve the rate of photosynthesis, chlorophyll content, transpiration rate and stomatal conductance in cucumber seedlings (Fan et al., 2007). In contrast, $\mathrm{Ni}$ stress caused a significant to extremely significant decrease in the photosynthetic pigment content. Decreases in chlorophyll contents under $\mathrm{Cu}$ stress were reported in Atriplex halimus (Brahim and Mohamed, 2011) and mangrove plant seedlings (Zhang et al., 2007). Exogenous applications of NO alleviate the inhibitory effects of Ni toxicity on the leaf pigment content. Similarly, Chen et al. (2010) detected that the NO-mediated stability and integrity of the subcellular structure under $\mathrm{Cd}$ stress contributed to its effective role in preventing $\mathrm{Cd}$-induced leaf chlorosis and in the inhibition of photosynthesis in barley seedlings. The detected increase in the photosynthetic pigments under $\mathrm{Ni}$ toxicity could be attributed to the role of NO in protecting chloroplast membranes from ROS or its involvement in the chlorophyll metabolic pathway (Siddiqui et al., 2013).

Based on the obtained results, SNP $(0.4 \mathrm{mM})$ has a stimulatory effect on the lupine carbohydrate content, including soluble sugars, polysaccharides and total carbohydrates. The soluble sugar content increases after an exogenous NO pre-treatment in wheat seedlings, which was in accordance with the increased amylase activity levels in the germinating seeds (Zheng et al., 2009). Ni stress significantly decreased the soluble sugar and total carbohydrate contents in lupine shoots and roots. Similarly, Latif (2010) demonstrated that a Ni sulfate treatment significantly decreases the total carbohydrate content of the white radish at moderate and higher concentrations $(0.178,0.36,0.53$ and $0.71 \mathrm{mM})$ compared with the untreated samples, but the red radish shows a significant decrease only at a higher concentration. Thus, the detected reduction in the photosynthetic pigment content had an indirect influence on the photosynthesis process and; therefore, the carbohydrate accumulation in plants. Heavy metals directly affect the photosynthetic machinery by binding to the various sensitive sites of the photosynthetic apparatus (Aggarwal et al., 2011). Interestingly, Ni-stressed plants treated with SNP $(0.4 \mathrm{mM})$ significantly increased the soluble sugar and polysaccharide contents compared with plants treated with 100 or $150 \mathrm{mM} \mathrm{Ni}$ sulfate alone. The increased accumulation of soluble sugar, which has been assigned an osmo-regulatory role, provides evidence for the development of water stress in $\mathrm{NiSO}_{4}$-treated plants (Najafi et al., 2011).

Based on the obtained results, SNP treatments decreased the proline contents in shoots and roots of lupine plants, while $\mathrm{NiSO}_{4}$ increased the contents when compared with untreated control plants. Similar results were obtained by Theriappan et al. (2011) who found that the applied concentrations of $\mathrm{ZnCl}_{2}$, $\mathrm{HgCl}_{2}$ and $\mathrm{CdCl}_{2}$ caused proline accumulation in cauliflower seedlings. Mourato et al. (2012) and Tripathi and Gaur (2004) proposed that ROS scavenging by proline, which is stimulated by heavy-metal stress, is primarily conducted by detoxifying hydroxyl radicals and quenching singlet oxygen. Proline can also act as a metal chelator and protein stabilizer (Mishra and Dubey, 2006). Proline accumulation in plant tissues has been attributed to the following: (1) an increase in proline biosynthesis; (2) a decrease in proline degradation; (3) a decrease in protein synthesis or proline use; and (4) the hydrolysis of proteins (Ashraf and Foolad, 2007). Further studies are needed to address the effects of NO and Ni stress on proline biosynthetic and degradative enzymes. The detected amounts of proline in plants treated with SNP $+\mathrm{Ni}$ sulfate were significantly lower than those treated with $\mathrm{Ni}$ alone. Similarly, Mihailovic and Drazic (2011) found that NO supplementation caused the proline accumulation stimulated by $\mathrm{Ni}$ to be completely suppressed in roots and partly suppressed in leaves.

Exogenous applications of NO significantly increased citric, malic, succinic, formic, acetic, fumaric and butyric acids in lupine plants, compared with in the control. Additionally, oxalic, citric, maleic, acetic and butyric acids were increased in both shoots and roots in response to all of the applied $\mathrm{Ni}$ sulfate concentrations, as shown in Figure 1. Organic acids not only act as intermediates in carbon metabolism but also as key components in coping mechanisms used by some plants in metal tolerance. The increased organic acid content may be a defense mechanism against $\mathrm{Ni}$ stress. At least two organic acids of the Krebs cycle, malic and citric, play important roles in metal-chelating processes by forming complexes with $\mathrm{Ni}$ (Brooks et al., 1991). Ni may be escorted to the vacuoles in the form of metal-organic acid complexes (Krämer et al., 2007).

Exogenous applications of an NO donor (0.4 mM SNP) highly significantly increased the $\mathrm{Mg}^{2+}$ and $\mathrm{Ca}^{2+}$ contents in lupine shoots and roots compared with untreated plants, while the $\mathrm{Ni}$ sulfate treatment highly significantly decreased the $\mathrm{Mg}^{2+}$ content compared with control plants. Exogenous NO alleviates the impact of $\mathrm{Ni}$ on the $\mathrm{Mg}^{2+}$ concentration, which may result from the activation of weakly selective Ca-uptake channels or increased requirements for $\mathrm{Mg}^{2+}$ by the NOactivated synthesis of enzymes and/or other proteins. Ni stress increased the accumulation of $\mathrm{Ca}^{2+}$ in lupine plants, and NO treatments caused additional $\mathrm{Ca}^{2+}$ accumulations compared with plants treated with $\mathrm{Ni}$ alone. Thus, $\mathrm{NO}$ responses to stresses in plants are mediated by a cytosolic $\mathrm{Ca}^{2+}$ increase. NO stimulates $\mathrm{Ca}^{2+}$ release from intracellular stores (Sokolowski and Blatt, 2007) but can also act as a strong stimulator of a $\mathrm{Ca}^{2+}$ influx across the plasma membrane (Lamotte et al., 2006). The mechanism involved is assumed to be a direct covalent 
modification of ion channels by the S-nitrosylation of proteins (Vandelle et al., 2006). The high accumulation level of $\mathrm{Ca}^{2+}$ in Ni-treated plants is probably necessary by the increased mobilization of intracellular and extracellular $\mathrm{Ca}^{2+}$ as mediators in the NO-regulated protective responses of the plant cells. $\mathrm{Ni}$ stress significantly increased endogenous $\mathrm{Ni}$ accumulations in lupine shoots and roots, while the NO treatment decreased the accumulated $\mathrm{Ni}$ amounts compared with untreated $\mathrm{Ni}$ stressed plants (Table 7). This might be attributed to the detected NO-stimulated organic acids, the formation of organic acid-metal complexes or to $\mathrm{Ni}$ accumulations in root cell wall, which subsequently decreased its levels in the aerial parts of the plant (Xiong et al., 2009).

NO treatment decreased the oxidative markers in lupine plants grown under normal or Ni-stress conditions (Table 8). SNP applications promoted ROS-scavenging enzymes, reduced $\mathrm{H}_{2} \mathrm{O}_{2}$ accumulation, increased the activity levels of $\mathrm{H}^{+}$-ATPase and $\mathrm{H}^{+}$-PPase in the plasma membrane or tonoplasts, and also significantly alleviated the growth inhibition induced by $\mathrm{CuCl}_{2}$ in tomato plants (Cui et al., 2009). Heavy metals cause cell toxicity by overproducing ROS, which impairs antioxidant defense systems and causes oxidative stress (Rui et al., 2016).

$\mathrm{NO}$ is most likely involved in $\mathrm{H}_{2} \mathrm{O}_{2}$ detoxification and in the maintenance of the cellular redox status (Tewari et al., 2008). SNP-exposed wheat plants show decreases in $\mathrm{H}_{2} \mathrm{O}_{2}$ and MDA under $\mathrm{Al}^{3+}$ stress (Zhang et al., 2008). NO protects the plants from oxidative damage by regulating the general mechanisms for cellular redox homeostasis, promoting the transformation of $\mathrm{O}_{2}^{-}$to $\mathrm{H}_{2} \mathrm{O}_{2}$ and $\mathrm{O}_{2}$, and enhancing the activity levels of $\mathrm{H}_{2} \mathrm{O}_{2}$-scavenging enzymes (Lamattina et al., 2003; Shi et al., 2007; Zheng et al., 2009).

\section{Materials and Methods}

\section{Plant materials}

Seeds of lupine (Lupinus termis L. cv. Balady) were obtained from Agriculture Research Center, Ministry of Agriculture, Giza, Egypt.

\section{Growth conditions}

This experiment was performed in the greenhouse of the National Institute of Occupational Safety and Health (NIOSH) in 2014 / 2015. Uniform seeds of Lupinus termis L. were surface sterilized with $2.5 \%$ sodium hypochlorite for $15 \mathrm{~min}$ and washed thoroughly with distilled water. 10 seeds were sown per plastic pot (22-cm diameter and $20-\mathrm{cm}$ length). Each pot was filled with $4 \mathrm{~kg}$ soil (clay: sand, 2:1 w/w). The plants were irrigated using tap water to $80 \%$ of their saturation capacity, and then $14 \mathrm{~d}$ after sowing; seedlings were thinned to 6 healthy seedlings per pot.

Three weeks after sowing, the pots were divided into three groups. The pots of the first group were sprayed with tap water to serve as a control; the second and third groups were sprayed two times (at $23 \mathrm{~d}$ and $30 \mathrm{~d}$ after sowing) with two concentrations of sodium nitroprusside $(0.4$ and $0.6 \mathrm{mM}$, respectively) as a nitric oxide (NO) donor. Each group was divided into three subgroups treated with one of the following concentrations of nickel sulfate: 0,100 and $150 \mathrm{mM}$. Samples were taken from 2-month-old plants to measure lipid peroxidation, $\mathrm{H}_{2} \mathrm{O}_{2}$, electrolyte leakage, growth parameters, photosynthetic pigments (including chlorophyll $a, b$ and carotenoids), endogenous phytohormones (IAA, GA,$A B A$ and $J A)$, soluble sugar and polysaccharide contents, proline, organic acids content and divalent cation $\mathrm{Ca}^{2+}, \mathrm{Mg}^{2+}$ and $\mathrm{Ni}^{2+}$ accumulations. The endogenous $\mathrm{NO}$ content in each treatment was also determined. Five planted pots from each level of treatment were considered.

\section{Estimation of endogenous NO}

Nitric oxide (NO) was detected using Griess reagent $(1 \%$ sulphanilamide, $0.1 \%$ naphthyethylene diamine dihydrochloride in $2 \% \mathrm{H}_{3} \mathrm{PO}_{3}$ ) as described by Sun et al. (2003). Results are expressed as $\mathrm{Hg} \mathrm{g}^{-1}$ fresh weight (FW).

Extraction, separation and estimation of growth regulating substances using gas chromatography (GC)

The method of extraction was similar to that adopted by Shindy and Smith (1975) and described by Hashem (2006). To estimate the amounts of acidic hormones IAA, $A B A$ and $G_{3}$, the plant hormone fractions and standards were methylated according to Vogel (1975). JA was determined according to Kramell (1996) using a NUCLEODEX beta-PM, $200 \mathrm{~mm} \times 4 \mathrm{~mm}$ ID column. The flow rate was adjusted to $1 \mathrm{ml} / \mathrm{min}$ and detected at UV $210 \mathrm{~nm}$. Results are expressed as ppm.

\section{Estimation of photosynthetic pigments}

The photosynthetic pigments (chlorophyll a, chlorophyll b and carotenoids) were determined in leaves of the investigated plants according to the spectrophotometric method described by Metzner et al. (1965). Pigment contents were calculated as $\mu \mathrm{g} \mathrm{g}^{-1} \mathrm{FW}$ of leaves.

\section{Estimation of carbohydrates}

Soluble sugars and polysaccharides were determined in plant tissues using anthrone method described by Whistler et al. (1962). Soluble sugars and polysaccharides were expressed as mg $100 \mathrm{~g}^{-1}$ DW.

\section{Estimation of proline}

Proline was determined using ninhydrin reagent (1.25 g ninhydrin in $30 \mathrm{ml}$ glacial acetic acid and $20 \mathrm{ml} 6 \mathrm{M}$ phosphoric acid) according to Bates et al. (1973). Results expressed as mg proline $100 \mathrm{~g}^{-1}$ DW. Griess Reaction assay was described by Bratton and Marshall in 1939.

\section{Estimation of organic acids}

An HP 1090 Hewlett-Packard liquid chromatograph equipped with a $\mathrm{Cl} 8$ guard column $(25 \times 4.6 \mathrm{~mm}$ I.D.), a column-switching valve, a Nucleosil ODS 100-5 analytical column $(250 \times 4.6 \mathrm{~mm}$ I.D.) and an auto sampler were used. UV-detection at $210 \mathrm{~nm}$ was accomplished with the HP 1040 diode-array detector. 
Organic acids were analyzed as described by Wodecki et al (1991).

\section{Estimation of mineral ions content and nickel accumulation}

The contents of $\mathrm{Ca}^{2+}, \mathrm{Mg}^{2+}$ and $\mathrm{Ni}^{2+}$ were analyzed using atomic absorption spectrometry (flame atomizer technique) (Marounek et al., 2006).

\section{Oxidative markers}

\section{Hydrogen peroxide content}

The hydrogen peroxide content was determined according to Shi et al. (2007) using 3\% (w/v) trichloroacetic acid, and the absorbance was measured at $390 \mathrm{~nm}$ (Alexieva et al., 2001). Data was expressed as $\mathrm{mM} \mathrm{H}_{2} \mathrm{O}_{2} \mathrm{~g}^{-1} \mathrm{FW}$ of plant.

\section{Lipid peroxidation}

The level of lipid peroxidation was measured in terms of malondialdehyde (MDA) content using the method of Hodges et al. (1999). The MDA content was calculated using its absorption coefficient of $155 \mathrm{mM}^{-1} \mathrm{~cm}^{-1}$ and expressed as $\mathrm{mM}$ $\mathrm{MDA} \mathrm{g}^{-1} \mathrm{FW}$.

\section{Electrolyte leakage}

Electrolyte leakage (EL) was determined according to DionisioSese and Torbita (1998).

\section{Statistical analysis}

Experiments utilized a completely randomized design. Mean values were calculated from the measurements of five replicates, and standard deviations of the means were analyzed using an independent sample t-test (SPSS program 17.0). To determine significant difference between means, two-tailed P-values were obtained (Snedecor and Cochran, 1980).

\section{Conclusion}

The present study showed that Ni toxicity can be ameliorated by an exogenous NO donor, as indicated by increased growth parameters, an enhanced photosynthetic pigment content and decreased oxidative-stress markers in $\mathrm{Ni}$-stressed plants treated with NO, compared with untreated plants subjected to the same $\mathrm{Ni}$ levels. Such effects could be attributed to the detected NO-enhanced hormonal levels, organic acid production and increased divalent cation $\left(\mathrm{Ca}^{2+}\right.$ and $\left.\mathrm{Mg}^{2+}\right)$ content. The extent of the impact depends on the NO concentration applied. A lower SNP concentration $(0.4 \mathrm{mM})$ alleviated the deleterious effects of up to $150 \mathrm{mM} \mathrm{Ni}$ sulfate, while a higher SNP concentration $(0.6 \mathrm{mM})$ alleviated the harmful effects of up to $100 \mathrm{mM}$ Ni sulfate.

\section{References}

Aggrawal A, Sharma I, Tripathi BN, Munjal AK, Baunthiyal M, Sharma V (2011) Metal toxicity and photosynthesis. Metal Toxicity and Photosynthesis. In: Shigeru et al. (eds) Photosynthesis: Overviews on Recent Progress \& Future Perspective, 1st edn. IK International Publishing House. New Delhi, 304 pages.

Alexieva V, Sergiev I, Mapelli S, Karanov E (2001) The effect of drought and ultraviolet radiation on growth and stress markers in pea and wheat. Plant cell Environ. 24:1337-1344.

Alaoui-Sossé B, Genet P, Vinit-Dunand F, Toussaint ML, Epron D, Badot PM (2004) Effect of copper on growth in cucumber plants (Cucumis sativus) and its relationships with carbohydrate accumulation and changes in ion contents. Plant Sci. 166: 1213-1218.

Ashraf M, Foolad MR (2007) Roles of glycine betaine and proline in improving plant abiotic stress resistance. Environ Exp Bot. 59:206216.

Atici Ö, Agar G, Battal P (2005) Changes in phytohormone contents in chickpea seeds germinating under lead or zinc stress. Biol Plant. 49:215-222.

Bates LS, Waldren RP, Teare ID (1973) Rapid determination of free proline for water stress studies. Plant Soil 39: 205-207.

Bethke PC, Libourel IGL, Aoyama N, Chung Y, Still DW and Jones $\mathrm{RL}(2007)$ The Arabidopsis thaliana aleurone layer responds to nitric oxide, gibberellin, and abscisic acid and is sufficient and necessary for seed dormancy. Plant Physiol. 143:1173-1188.

Bethke PC, Libourel IGL, Jones RL (2006) Nitric oxide reduces seed dormancy in Arabidopsis. J Exp Bot. 57:517-526.

Beligni MV, Lamattina L (2001) Nitric oxide in plants: the history is just beginning. Plant Cell Environ. 24:267-278.

Beligni MV, Lamattina L (2000) Nitric oxide stimulates seed germination and de-etiolation, and inhibits hypocotyl elongation, three light-inducible responses in plants. Planta 210:215-221.

Bright J, Desikan R, Hancock JT, Weir IS, Neill SJ (2006) ABA induced NO generation and stomatal closure in Arabidopsis are dependent on $\mathrm{H}_{2} \mathrm{O}_{2}$ synthesis. Plant J. 45:113-122.

Brooks R, Shaw S, Marfil AA (1981) The chemical form and physiological function of nickel in some Iberian Alyssum species. Physiol Plant. 51:167-170

Chen F, Wang F, Sun HY, Cai Y, Mao WH, Zhang GP, Vincze E, Wu FB ( 2010): Genotype-dependent effect of exogenous nitric oxide on Cdinduced changes in antioxidative metabolism, ultrastructure, and photosynthetic performance in barley seedlings (Hordeum vulgare). J Plant Growth Regul. 29:394-408.

Cui X, Zhang Y, Chen X, Jin H, Wu X (2009) Effects of exogenous nitric oxide protects tomato plants under copper stress. Bioinformatics and Biomedical Engineering, 2009. ICBBE 2009. 3rd International Conference on 11-13 June 2009, Beijing, p 1-7.

Desikan R, Cheung MK, Bright J, Henson D, Hancock JT, Neill SJ (2004) ABA, hydrogen peroxide and nitric oxide signaling in stomatal guard cells. J Exp Bot. 55: 205-212.

Besson-Bard A, Gravot A, Richaud P, Auroy P, Taconnat L, Renou J, Pugin A, Wendehenne D (2009) Nitric oxide contributes to cadmium toxicity in Arabidopsis by promoting cadmium accumulation in roots and by up-regulating genes related to iron uptake. Plant Physiol. 149:1302-1315.

Brahim L, Mohamed M (2011): Effects of copper stress on antioxidative enzymes, chlorophyll and protein content in Atriplex halimus. Afr J Biotechnol. 50:10143-10148.

Dionisio-Sese MI, Tobita S (1998) Antioxidant responses of rice seedlings to salinity stress. Plant Sci. 135: 1-9.

Fan H, Guo S, Jiao Y, Zhang R, Li J (2007) Effects of exogenous nitric oxide on growth, active oxygen species metabolism, and photosynthetic characteristics in cucumber seedlings under $\mathrm{NaCl}$ stress. Front Agr China 1 (3): 308-314. 
Feng J, Wang C, Chen Q, Chen H, Ren B, Li X, Zuo J (2013) Snitrosylation of phosphotransfer proteins represses cytokinin signaling. Nat Commun. 4:1529.

Floryszak-Wieczorek J, Arasimowicz M, Milczarek G, Jelen $H$, Jackowiak $H$ (2007) Only an early nitric oxide burst and the following wave of secondary nitric oxide generation enhanced effective defense responses of pelargonium to a necrotrophic pathogen. New Phytol. 175: 718-730.

Freschi L (2013) Nitric oxide and phytohormone interactions: current status and perspectives. Front Plant Sci. 4: 398.

Gajewska E, Sklodowska M (2005) Antioxidative responses and proline level in leaves and roots of pea plants subjected to nickel stress. Acta Physiol Plant 27: 329-339.

Gallego SM, Kogan MJ, Azpilicueta CE, Pe na C, Tomaro ML (2005) Glutathione-mediated antioxidative mechanisms in sunflower (Helianthus annuus L.) cells in response to cadmium stress. Plant Growth Regul. 46: 67-276.

García-Mata C, Lamattina L (2007) Abscisic acid (ABA) inhibits lightinduced stomatal opening through calcium- and nitric oxidemediated signaling pathways. Nitric Oxide 17:143-151.

García-Mata C, Lamattina L. ( 2002) Nitric oxide and abscisic acid cross talk in guard cells. Plant Physiol. 128: 790-792.

García-Mata C, Lamattina L. (2001): Nitric oxide induces stomatal closure and enhances the adaptive plant responses against drought stress. Plant Physiol. 126:1196-1204

Grossi L, D’ Angelo S (2005) Sodium Nitroprusside: Mechanism of NO Release Mediated by Sulfhydryl-Containing Molecules. J Med Chem. 48 (7): 2622-2626.

Groppa MD, Rosales EP, lannone MF, Benavides MP (2008) Nitric oxide, polyamines and $\mathrm{Cd}$-induced phytotoxicity in wheat roots. Phytochem. 69: 2609-2915.

Guo FQ, Crawford NM (2005) Arabidopsis nitric oxide synthase1 is targeted to mitochondria and protects against oxidative damage and dark-induced senescence. Plant Cell 17:3436-3450.

Guo FQ, Okamoto M, Crawford NM (2003) Identification of a plant nitric oxide synthase gene involved in hormonal signaling. Sci. 302:100-103.

Hashem HA (2006) Physiological and molecular actions of jasmonic acid on soybean plant. Ph.D. Thesis, Fac. of Sci., Ain Shams Univ., Cairo, Egypt.

He YK, Tang RH, Yi H, Stevens RD, Cook CW, Ahn SM, Jing L, Yang Z, Chen L, Guo F, Fiorani F, Jackson RB, Crawford NM, Pei ZM (2004) Nitric oxide represses the Arabidopsis floral transition. Sci. 305:1968-1971.

Hodges DM, DeLong JM, Forney CF, Prange RK (1999) Improving the thiobarbituric acid-reactive-substances assay for estimating lipid peroxidation in plant tissues containing anthocyanin and other interfering compounds. Planta 207:604-611.

Hu X, Neill SJ, Tang Z, Cai W (2005) Nitric oxide mediates gravitropic bending in soybean roots. Plant Physiol. 137:663-670.

Hyang LE, Dae KH, Seung KL (2009) Nitric Oxide Reduced Chlorophyll Degradation in Broccoli (Brassica oleracea L. var. italica) Florets During Senescence. Food Sci Tech Inter. 15 (3): 223-228.

Kopyra M, Gwóźdź E (2003) Nitric oxide stimulates seed germination and counteracts the inhibitory effect of heavy metals and salinity on root growth of Lupinus luteus. Plant Physiol Biochem. 41: 10111017.

Kramell R (1996) HPLC separation of jasmonic acid methyl ester enantiomers. Phytochem analysis 7: 738-743.

Krämer U, Talke IN, Hanikenne M (2007) Transition metal transport. FEBS Lett. 581, 2263-2272.

Lamattina L, Garcia-Mata C, Graziano M, Pagnussat G (2003) Nitric oxide: the versatility of an extensive signal molecule. Annu Rev Plant Biol. 54:109-136.

Lamotte O, Courtois C, Dobrowolska G, Besson A, Pugin A, Wendehenne D (2006) Mechanisms of nitric oxide-induced increase of free cytosolic $\mathrm{Ca}^{2+}$-concentration in Nicotiana plumbaginifolia cells. Free Radical Biol Med. 40: 1369-1376.
Laspina NV, Groppa MD, Tomaro ML, Benavides MP (2005) Nitric oxide protects sunflower leaves against $\mathrm{Cd}$-induced oxidative stress. Plant Sci 169: 323-330.

Latif HH (2010) The influence of nickel sulphate on some physiological aspects of two cultivars of Raphanus sativus L. Arch Biol Sci Belgrade 62:683-691.

Leon J, Lozano-Juste J (2011) Nitric oxide regulates DELLA content and PIF expression to promote photomorphogenesis in Arabidopsis. Plant Physiol 156 1410-1423.

Libourel IGL, van Bodegom PM, Fricker MD, Ratcliffe RG (2006) Nitrite reduces cytoplasmic acidosis under anoxia. Plant Physiol. 142:17101717.

Lin $\mathrm{CH}$, Chen LM, Liu ZH (2005) Rapid effect of copper on lignin biosynthesis in soybean roots. Plant Sci. 168: 855-861.

Maksymiec W (2007) Signaling responses in plants to heavy metal stress. Acta Physiol. Plant. 29: 177-187.

Marounek M, Skřivanová E, Skřivanová V (2006)Selenium content and antioxidant status in tissues of veal calves fed a diet supplemented with selenium yeast. Slovak J Anim Sci. 39(1-2): 51-54.

Metzner H, Rau H, Senger H (1965) Untersuchungen Zur Synchronisier barkeep ein Zelner pigment. Mango I Mutanten Von chlorella. Planta 65: 186.

Mihailovic N, Drazic G (2011) Incomplete alleviation of nickel toxicity in bean by nitric oxide supplementation. Plant Soil Environ. 57:396401.

S. Mishra and R. S. Dubey (2006) Heavy metal uptake and detoxification mechanisms in plants. Inter J Agri Res. 1 (2): 122141.

Modolo LV, Augusto O, Almeida IM, Magalhaes JR, Salgado I. et al. (2005): Nitrite as the major source of nitric oxide production by Arabidopsis thaliana in response to Pseudomonas syringae. FEBS Lett 579: 3814-3820.

Molas J (2002) Changes of chloroplast ultrastructure and total chlorophyll concentration in cabbage leaves caused by excess of organic Ni (II) complexes. Environ Exp Bot. 47: 115-126.

Monni S, Uhlig C, Hansen E, Magel E (2001) Ecophysiological responses of Empetrum nigrum to heavy metal pollution. Environ Pollut. 112(2):121-9.

M. Mourato, Reis R, Martins LL (2012) Characterization of plant antioxidative system in response to abiotic stresses: a focus on heavy metal toxicity. In: Montanaro G, Dichio B (eds), Advances in Selected Plant Physiology Aspects. In Tech, Vienna, Austria, p 23-44.

Mukhopadhyay M, Mondal TK (2015) Effect of zinc and boron on growth and water relations of Camellia sinensis (L.) O. Kuntze cv. T78. Natl Acad Sci Lett 38:283-286.

Najafi F, Khavari-Nejad RA, Hasanjanzadeh F (2011) The Physiological responses of sunflower (Helianthus annuus L.) to $\mathrm{NiSO}_{4}$ Afr J Plant Sci 5 (3): 201-206

Neill S, Desikan R, Hancock JT (2003) Nitric oxide signaling in plants. New Phytol 159:11-35.

Pantin F, Monnet F, Jannaud D, Costa JM, Renaud J, Muller B Simonneau T, Genty B (2013) The dual effect of abscisic acid on stomata. New Phytol. 197:65-72.

Parani M, Rudrabhatla S, Myers R, Weirich H, Smith B, Leaman DW et al. (2004) Microarray analysis of nitric oxide responsive transcripts in Arabidopsis. Plant Biotechnol J. 2: 359-366. doi: 10.1111/j.14677652.2004.00085.x

Pedroso MC, Durzan DJ (2000) Effect of different gravity environments on DNA fragmentation and cell death in Kalanchoe leaves. Ann Bot 86:983-994.

Rascio N, Navari-Izzo F ( 2011) Heavy metal hyper-accumulating plants: "how and why do they do it? And what makes them so interesting?" Plant Sci. 180(2): 169-181.

Rui H, Chen C, Zhang X, Shen Z, Zhang F (2016) Cd-induced oxidative stress and lignification in the roots of two Vicia sativa L. varieties with different Cd tolerances. J Hazard Mater. 301:304-313. 
Sakihama Y, Murakami S Yamasaki H (2003) Involvement of nitric oxide in the mechanism for stomatal opening in Vicia faba leaves. Biol Plant 46:117-119.

Shi Q, Ding F, Wang X, Wei M (2007): Exogenous nitric oxide protects cucumber roots against oxidative stress induced by salt stress. Plant Physiol Biochem. 45:542-550.

Shindy WW, Smith O (1975) Identification of plant hormones from cotton ovules. Plant Physiol. 55: 550-554.

Siddiqui MH , Al-Whaibi MH, Ali HM, Sakran AM, Basalah MO, Al Khaishany MYY (2013) Mitigation of nickel stress by the exogenous application of salicylic acid and nitric oxide in wheat . Aust J crop Sci 7(11):1780-1788.

Snedecor GW, Cochran WG (1980) Statistical methods. $7^{\text {th }}$ edn, lowa State University Press, Ames, lowa.

Sobkowiak R Dekert J (2003) Cadmium induced changes in growth and cell cycle gene expression in suspension-culture cells of soybean. Plant Physiol Biochem. 41: 767-772.

Sokolovski SG, Blatt MR (2007) Nitric oxide and plant ion channel control. In: Lamattina L, Polacco JC (eds), Nitric Oxide in Plant Growth, Development and Stress Physiology. Springer Berlin, Heidelberg, p153-171.

Srivastava S, Chiappetta A, Beatrice M (2013) Identification and profiling of arsenic stress-induced miRNAs in Brassica juncea. J Exp Bot. 64:303-315.

Sun J, Zhang X, Broderick M, Fein H. (2003): Measurement of Nitric Oxide Production in Biological Systems by Using Griess Reaction Assay. Sensors 3, 276-284.

Takahashi S, Yamasaki H (2002) Reversible inhibition of photophosphorylation in chloroplasts by nitric oxide. FEBS Lett 512:145-148.

Teisseire H GuyV ( 2000) Copper-induced changes in antioxidant enzymes activities in fronds of duckweed (Lemna minor). Plant Sci. 153: $65-72$.

Terrile MC, París R, Calderón-Villalobos LI, Iglesias MJ, Lamattina L, Estelle M, Casalongué CA (2012) Nitric oxide influences auxin signaling through S-nitrosylation of the Arabidopsis TRANSPORT INHIBITOR RESPONSE 1 auxin receptor. Plant J. 70(3):492-500.

Tewari RK, Hahn EJ, Paek KY (2008) Modulation of copper toxicity induced oxidative damage by nitric oxide supply in the adventitious roots of Panax ginseng. Plant Cell Rep 27:171-181.

Theriappan P, Gupta AK, Dhasarathan P (2011) Accumulation of Proline under Salinity and Heavy metal stress in Cauliflower seedlings. J Appl Sci Environ Manage. 15 (2) 251 - 255

Tian X, Lei Y (2006) Nitric oxide treatment alleviates drought stress in wheat seedlings. Biol Plant. 50: 775-778.

Tripathi BN, Gaur JP (2004) Relationship between copper- and zincinduced oxidative stress and proline accumulation in Scenedesmus sp. Planta 219 (3)397-404.

Uchida A, Jagendorf AT, Hibino T, Takabe T (2002) Effects of hydrogen peroxide and nitric oxide on both salt and heat stress tolerance in rice. Plant Sci. 163: 515-523.

Valentovičová K, Halušková L, Huttová J, Mistrík I, Tamás L (2010) Effect of cadmium diaphorase activity and nitric oxide production in barley root tips. J Plant Physiol. 167: 10-14.

Vandelle E, Poinssot B, Wendehenne D, Bentejac M, Pugin A (2006) Integrated signaling network involving calcium nitric oxide, active oxygen species but not mitogen-activated protein kinases in BcPG1elicited grapevine defenses. Mol Plant Microb Interact. 19: 429-440.
Villiers F, Ducruix C, Hugouvieux V et al (2011) Investigating the plant response to cadmium exposure by proteomic and metabolomic approaches. Proteomics 11(9):1650-1663.

Vogel AJ (1975) A Text Book of Practical Organic Chemistry, 3rd edn, English Language Book Society and Longman Group Ltd. p 483-485.

Wendehenne D, Durner J, Klessig DF (2004) Nitric oxide: a new player in plant signaling and defense responses. Curr Opin Plant Biol. 7: 449-455.

Wendehenne D, Pugin A, Klessig DF, Durner J (2001) Nitric oxide: comparative synthesis and signaling in animal and plant cells. Trends Plant Sci. 6: 177-183.

Whistler RL, Wolform ML, BeMiller JN, Shafizadeh F (1962) Anthrone colorimetric method. In: methods in carbohydrate chemistry. Academic Press, New York, London, 1: 384.

Wodecki ZJ, Torłop B, Ślebioda M (1991) Chromatographic determination of citric acid for monitoring the mold process. J Chromatography A 558:302-5.

Xu J, Wang WY, Yin HX, Liu XJ, Sun H, Mi Q (2010) Exogenous nitric oxide improves antioxidative capacity and reduces auxin degradation in roots of Medicago truncatula seedlings under cadmium stress. Plant Soil 326: 321-330 10.1007/s11104-009-0011-

Zanardo DIL, Zanardo FML, Ferrarese MLL, Magalhaes JR, FerrareseFilho $O$ (2005) Nitric oxide affecting seed germination and peroxidase activity in canola (Brassica napus. L.). Physiol Mol Biol Plants 11: 81-86.

Zelinová V, Alemayehu A, Bocová B, Huttová J, Tamás L (2015) Cadmium-induced reactive oxygen species generation, changes in morphogenic responses and activity of some enzymes in barley root tip are regulated by auxin. Biologia 70:356-364.

Zemojtel T, Fröhlich T, Palmieri MC, Kolanczyk M, Mikula I, Wyrwicz LS, Wanker EE, Mundlos S, Vingron M, Martasek P, Durner J (2006) Plant nitric oxide synthase: a never-ending story? Trends Plant Sci. 11:524-525.

Zhang H, Li YH, Hu LY, Wang SH, et al (2008) Effects of exogenous nitric oxide donor on antioxidant metabolism in wheat leaves under aluminum stress. J Plant Physiol. 55: 469-474.

Zhang FQ, Wang YS, Lou ZP, Dong GD (2007) Effect of heavy metal stress on antioxidative enzymes and lipid peroxidation in leaves and roots of two mangrove plant seedlings (Kandelia candel and Bruguiera gymnorrhiza). Chemotherapy 67:44-50.

Zhang L, Wang Y, Zhao L, Shi S, Zhang L et al. (2006a) Involvement of nitric oxide in light-mediated greening of barley seedlings. J Plant Physiol. 163:818-826.

Zhang YY, Wang LL, Liu YL, Zhang Q, Wei QP, Zhang WH (2006b) Nitric oxide enhances salt tolerance in maize seedlings through increasing activities of proton-pump and $\mathrm{Na}^{+} / \mathrm{H}^{+}$antiport in the tonoplast. Planta 224:545-555.

Zhao MG, Tian QY, Zhang WH (2007) Nitric oxide synthase-dependent nitric oxide production is associated with salt tolerance in Arabidopsis. Plant Physiol. 144:206-217.

Zheng C, Jiang D, Liu F, Dai T, Liu W, Jing Q, Cao W (2009) Exogenous nitric oxide improves seed germination in wheat against mitochondrial oxidative damage induced by high salinity. Environ Exp Bot. 67:222-227.

Zottini M, Formentin E, Scattolin M, Carimi F, Schiavo L, Terzi M (2002) Nitric oxide affects plant mitochondrial functionality in vivo. FEBS Lett 515: 75-78. 